\title{
Skyrme-force time-dependent Hartree-Fock calculations with axial symmetry
}

\author{
K. T. R. Davies \\ Physics Division, Oak Ridge National Laboratory, Oak Ridge, Tennessee 37830 \\ S. E. Koonin \\ W. K. Kellogg Radiation Laboratory, California Institute of Technology, Pasadena, California 91125 \\ (Received 14 October 1980)
}

\begin{abstract}
We discuss axially symmetric time-dependent Hartree-Fock calculations using a finite-range modification of the Skyrme energy functional. The finite-difference forms of the coordinate-space time-dependent Hartree-Fock equations, the method of time evolution, and other numerical aspects are presented. Detailed results for ${ }^{84} \mathrm{Kr}$ induced deep-inelastic collisions with ${ }^{208} \mathrm{~Pb}$ at $E_{\mathrm{lab}}=494 \mathrm{MeV}$ and with ${ }^{209} \mathrm{Bi}$ at $E_{\mathrm{lab}}=600 \mathrm{MeV}$ and $714 \mathrm{MeV}$ are compared with experiment.
\end{abstract}

$\left[\begin{array}{l}\text { NUCLEAR REACTIONS }{ }^{84} \mathrm{Kr}+{ }^{208} \mathrm{~Pb} \text { at } E_{\text {lab }}=494 \mathrm{MeV} \text { and }{ }^{84} \mathrm{Kr}+{ }^{209} \mathrm{Bi} \text { at } E_{\text {lab }} \\ =600 \text { and } 714 \mathrm{MeV} \text {, in the time-dependent Hartree-Fock approximation. Strong- } \\ \text { ly damped collisions. Details of Skyrme force calculations with axial symmetry. }\end{array}\right]$

\section{INTRODUCTION}

Time-dependent Hartree-Fock (TDHF) calculations are an attempt to microscopically describe the dynamics of large nuclear systems, e.g., heavy-ion collisions and fission. Their fundamental physical assumption is that the well-established independent particle behavior for near-equilibrium nuclear states persists to highly nonequilibrium situations if the excitation energy per nucleon is less than several MeV. While this assumption is a priori plausible, it can only be tested and refined by a systematic comparison between the results of realistic TDHF calculations and experimental data. This paper describes several of the steps we have taken toward achieving this objective.

Following the first schematic applications of TDHF to large amplitude nuclear dynamics, ${ }^{1-4}$ a sequence of calculations has appeared ${ }^{5-34}$ in which the geometrical and isospin symmetries of the determinantal wave function have been progressively relaxed and the effective interaction steadily improved. Calculations are now available which include one or more of the following: a nonlocal mean field (Refs. 8, 9, 14, 18-20, 23, 25, $26,28,29,33,34)$, a finite-range effective interaction (Refs. 6-9,11-15, 18-23, 25-34), the difference between neutrons and protons (Refs. 8, 9, 14, $18-20,22,23,25,26,28,29,33,34)$, the pairing force (Refs. 18, 21, 27, 30-32), mass asymmetry (Refs. 7, 8, 10, 19, 22, 23, 26, 28-34), and a complete (Refs. 5, 10-13, 15, 17, 21, 24, 27, 30-32), [or nearly complete (Refs. 15-17, 23, 24, 29, 33, 34)] three-dimensional geometry. A number of different physical situations have been studied: fis sion, ${ }^{18}$ heavy -ion fusion (Refs. $6,7,11,12,14,19$, $22,23,25,26,29,31,33,34)$, and deep-inelastic heavy-ion collisions (Refs. 5-11, 13, 15-17,
$19-21,24,25,27-30,32,33)$, with varying degrees of qualitative and even quantitative success. How ever, the complexity of these calculations often makes difficult (or occasionally precludes) any systematic study of the dependence of the results upon initial conditions or the effective interaction used.

We have two goals in the present work. First, over the last three years we have developed a computational technology for solving the TDHF equations in an axially symmetric geometry using coordinate space finite difference methods. These methods, which grew out of earlier, simpler calculations, ${ }^{2,6,7,13}$ use a finite-range modification $^{1,18,35}$ of a Skyrme-type effective interaction ${ }^{36}$ and can treat a nonlocal mean field, neutron-proton asymmetries, and mass-asymmetric systems. Although a number of studies based on these more sophisticated codes have been completed (Refs. $8,9,14,18-20,22,23,25,26,28)$, a detailed exposition of their methods and our experience in their use does not exist. Such an exposition, contained in this paper, seems particularly appropriate at the present time in view of the widespread use of these codes and the potential use of these or related methods in the application of mean-field theories $^{37-41}$ to nuclear ${ }^{42}$ and atomic ${ }^{43,44}$ problems.

Our second goal in writing this work is to present some systematic results for ${ }^{84} \mathrm{Kr}$-induced reactions on ${ }^{208} \mathrm{~Pb}$ and ${ }^{209} \mathrm{Bi}$ at three different bombarding energies ( $E_{1 \mathrm{ab}}=494,600$, and $\left.714 \mathrm{MeV}\right)$. Some results at the two lower energies have been published previously. ${ }^{8}$ For these three systems, we calculate the scattering angle, kinetic energy, charge, mass, and mass dispersion of the products as functions of the initial angular momentum and compare our results with both available experimental data ${ }^{45,46}$ and phenomenological expec- 
tations. ${ }^{47}$ The influence of some of the microscopic aspects of TDHF on the experimental observables is also discussed.

The philosophy and formalism of the TDHF method has been discussed in several articles, ${ }^{1,48-50}$ to which we refer the interested reader for background to the present paper. The exposition here concerns only the essential features of our particular implementation of TDHF for heavyion collisions. In Sec. II we discuss the effective interaction used and the corresponding energy functional. Section III details the spatial discreti- zation of the energy functional, while Sec. IV gives the resulting finite-difference forms of the TDHF equations. In Sec. $V$ we discuss the rotating frame approximation used to relate the axially symmetric geometry to three-dimensional collisions at a nonzero impact parameter. Section VI contains other details of the calculation such as initial conditions, the extraction of final-state quantities, and the parameters of the space-time mesh. Our results for the $\mathrm{Kr}$-induced collisions are presented in Sec. VII.

\section{THE ENERGY FUNCTIONAL}

Our calculations use a suitably modified Skyrme form for the nuclear interaction together with the Coulomb interaction. $1,18,35,36$ The total energy function for the system, $\mathcal{H}$, is thus

$$
\mathcal{H}=\mathcal{H}_{n}+\mathcal{H}_{C},
$$

where $\mathcal{F}_{n}$ is the nuclear energy and $\mathcal{F}_{C}$ is the Coulomb energy.

\section{A. The nuclear energy}

In constructing the nuclear energy, we neglect the presumably small effects due to the spin-orbit force and assume a spin-saturated system, so that each spatial orbital is occupied by two nucleons, "spin-up" and "spin-down." Such spin effects are unlikely to significantly influence the bulk nuclear dynamics of interest in this paper. However, we do distinguish between neutrons and protons, as is essential for a realistic treatment of the $N>Z$ systems we consider. Under these assumptions, the original Skyrme form of the nuclear energy, $\mathfrak{H}_{s}$, is ${ }^{36,51}$

$$
\begin{aligned}
\mathcal{H}_{s}=\int d \overline{\mathrm{F}} & \left\{\frac{\hbar^{2}}{2 m} \tau+\frac{1}{2} t_{0}\left[\left(1+\frac{x_{0}}{2}\right) \rho^{2}-\left(\frac{1}{2}+x_{0}\right) \sum_{q} \rho_{a}{ }^{2}\right]+\frac{t_{3}}{8}\left(\rho^{3}-\rho \sum_{q} \rho_{q}^{2}\right)\right. \\
& \left.+\frac{t_{1}+t_{2}}{4}\left(\rho \tau-j^{2}\right)+\frac{t_{2}-t_{1}}{8} \sum_{a}\left(\rho_{a} \tau_{a}-j_{q}{ }^{2}\right)+\frac{1}{16}\left(t_{2}-3 t_{1}\right) \rho \nabla^{2} \rho+\left(\frac{t_{2}+3 t_{1}}{32}\right) \sum_{a} \rho_{q} \nabla^{2} \rho_{a}\right\} .
\end{aligned}
$$

The particle, kinetic energy, and current densities for each isospin species $q$ ( $p$ for protons, $n$ for neutrons) are defined as

$$
\begin{aligned}
& \rho_{q}(\overrightarrow{\mathrm{r}})=\sum_{\alpha \in q}\left|\psi_{\alpha}(\overrightarrow{\mathrm{r}})\right|^{2}, \\
& \tau_{q}(\overrightarrow{\mathrm{r}})=\sum_{\alpha \in q}\left|\nabla \psi_{\alpha}(\overrightarrow{\mathrm{r}})\right|^{2}, \\
& \overrightarrow{\mathrm{j}}_{q}(\overrightarrow{\mathrm{r}})=\sum_{\alpha \in q} \operatorname{Im}\left[\psi_{\alpha}^{*}(\overrightarrow{\mathrm{r}}) \nabla \psi_{\alpha}(\overrightarrow{\mathrm{r}})\right],
\end{aligned}
$$

where the sums are over all occupied single-particle orbitals having isospin $q$. Densities without isospin subscripts refer to the total density (i.e., $\rho=\rho_{p}+\rho_{n}, \tau=\tau_{p}+\tau_{n}, \vec{j}=\vec{j}_{p}+\vec{j}_{n}$ ). The parameters $t_{0}, x_{0}, t_{3}, t_{1}$, and $t_{2}$ appearing in Eq. (2.2) are the usual constants of the Skyrme force. ${ }^{36}$ In our time-dependent calculations, all of the functions in Eq. (2.3) depend on time $t$ as well as on the spatial coordinates $\overrightarrow{\mathbf{r}}$. However, for convenience of notation, we henceforth suppress the dependence on $t$.

In order to simulate the effect of the finite range of the nuclear force, as well as to improve the stability of our finite-difference numerical calculations, we have found it convenient to replace the surface energy terms in (2.2) of the form $\rho \nabla^{2} \rho$ by the following sum of direct Yukawa interactions ${ }^{1,18,35}$ :

$$
\mathcal{H}_{y}=\int d \overrightarrow{\mathrm{r}} d \overrightarrow{\mathbf{r}}^{\prime} \frac{e^{-\left|\overrightarrow{\mathbf{r}}-\overrightarrow{\mathbf{r}}^{\prime}\right| / a}}{\left|\overrightarrow{\mathbf{r}}-\overrightarrow{\mathbf{r}}^{\prime}\right| / a}\left[\frac{V_{u}}{2} \rho(\overrightarrow{\mathrm{r}}) \rho\left(\overrightarrow{\mathbf{r}}^{\prime}\right)+\left(\frac{V_{l}-V_{u}}{2}\right) \sum_{a} \rho_{a}(\overrightarrow{\mathrm{r}}) \rho_{q}\left(\overrightarrow{\mathbf{r}}^{\prime}\right)\right],
$$

where $V_{l}$ and $V_{u}$ are the strengths of the interactions between "like" and "unlike" nucleons and $a$ is the range of the force. The connection between (2.4) and the $\rho \nabla^{2} \rho$ terms of (2.2) can readily be seen by a 
Taylor series expansion of the form

$$
\iint d \overrightarrow{\mathbf{r}} d \overrightarrow{\mathbf{r}}^{\prime} \frac{e^{-\left|\overrightarrow{\mathrm{r}}-\overrightarrow{\mathbf{r}}^{\prime}\right| / a}}{\left|\overrightarrow{\mathrm{r}}-\overrightarrow{\mathrm{r}}^{\prime}\right| / a} \rho_{q}(\overrightarrow{\mathrm{r}}) \rho_{q^{\prime}}\left(\overrightarrow{\mathrm{r}}^{\prime}\right)=4 \pi a^{3}\left\{\int d \overrightarrow{\mathbf{r}} \rho_{q}(\overrightarrow{\mathrm{r}}) \rho_{q^{\prime}}(\overrightarrow{\mathrm{r}})+\frac{1}{2} a^{2} \int d \overrightarrow{\mathrm{r}}\left[\rho_{q}(\overrightarrow{\mathrm{r}}) \nabla^{2} \rho_{q^{\prime}}(\overrightarrow{\mathrm{r}})+\rho_{q^{\prime}}(\overrightarrow{\mathrm{r}}) \nabla^{2} \rho_{q}(\overrightarrow{\mathrm{r}})\right]+\cdots\right\}
$$

Thus, (2.4) will approximately reproduce the surface terms of (2.2) if $V_{l}, V_{u}$, and $a$ are chosen to satisfy

$$
\begin{aligned}
& 2 \pi V_{u} a^{5}=\frac{1}{16}\left(t_{2}-3 t_{1}\right), \\
& 2 \pi\left(V_{l}-V_{u}\right) a^{5}=\frac{1}{32}\left(t_{2}+3 t_{1}\right) .
\end{aligned}
$$

In our calculations, we have chosen $a$ to approximate the range of the $G$ matrix in nuclear matter, ${ }^{1}$ so that Eqs. (2.6) determine $V_{u}$ and $V_{l}$. The presence of the zeroth order "volume" term in (2.5) also requires a renormalization of the parameters $t_{0}, x_{0}$ in (2.2) to $\tilde{t}_{0}, \tilde{x}_{0}$, satisfying

$$
\begin{aligned}
& \frac{1}{2} \tilde{t}_{0}\left(1+\tilde{x}_{0} / 2\right)=\frac{1}{2} t_{0}\left(1+x_{0} / 2\right)-2 \pi a^{3} V_{u}, \\
& \frac{1}{2} \tilde{t}_{0}\left(\frac{1}{2}+\tilde{x}_{0}\right)=\frac{1}{2} t_{0}\left(\frac{1}{2}+x_{0}\right)+2 \pi a^{3}\left(V_{l}-V_{u}\right) .
\end{aligned}
$$

These equations may also be used to obtain new values of $t_{0}$ and $x_{0}$ when one uses the zero range Skyrme force $\left(t_{1}=t_{2}=0\right)$ plus a direct Yukawa interaction as in Ref. 1.

Thus, in summary, we take the nuclear energy to be

$$
\begin{aligned}
\mathcal{H}_{n}=\int d \overrightarrow{\mathrm{r}} & \left\{\frac{\hbar^{2}}{2 m} \tau+\frac{1}{2} \tilde{t}_{0}\left[\left(1+\tilde{x}_{0} / 1\right) \rho^{2}-\left(\frac{1}{2}+\tilde{x}_{0}\right) \sum_{q} \rho_{q}{ }^{2}\right]\right. \\
& +\frac{t_{3}}{8}\left(\rho^{3}-\rho \sum_{q} \rho_{q}^{2}\right)+\frac{t_{1}+t_{2}}{4}\left(\rho \tau-j^{2}\right) \\
& \left.+\left(\frac{t_{2}-t_{1}}{8}\right) \sum_{q}\left(\rho_{q} \tau_{q}-j_{q}^{2}\right)\right\}+\mathcal{H}_{y} .
\end{aligned}
$$

Our calculations presented here employ the modified interaction derived from the Skyrme II force,$^{18,35,36}$ as given in Table I. With this force, nuclear matter saturates at a Fermi wave number of $k_{F}=1.30 \mathrm{fm}^{-1}$ (density $\rho=0.15 \mathrm{fm}^{-3}$ ) with a binding energy per nucleon of $+16.0 \mathrm{MeV}$, an incompressibility coefficient of about $340 \mathrm{MeV}$, and a symmetry energy coefficient of $34.1 \mathrm{MeV}$. The binding energies and $\mathrm{rms}$ radii calculated with this force for nuclei over a wide range of the periodic table are in good agreement with experiment. However, for light systems the surface energy for the modified force is somewhat smaller than that of the original Skyrme potential and some parameter readjustment may be necessary for future calculations. ${ }^{35}$

\section{B. The Coulomb energy}

The Coulomb contribution to the energy functional is taken to be

$$
\mathcal{H}_{C}=\mathcal{H}_{C}^{(\mathrm{dir} r)}+\mathcal{H}_{C}^{(\text {ex })},
$$

where the direct energy is

$$
\mathcal{F}_{C}^{(\mathrm{dir})}=\frac{1}{2} e^{2} \int d \overrightarrow{\mathrm{r}} d \overrightarrow{\mathrm{r}}^{\prime} \rho_{p}(\overrightarrow{\mathrm{r}}) \frac{1}{\left|\overrightarrow{\mathbf{r}}-\overrightarrow{\mathbf{r}}^{\prime}\right|} \rho_{p}\left(\overrightarrow{\mathrm{r}}^{\prime}\right),(2.10 \mathrm{a})
$$

and the Slater approximation to the exchange energy is ${ }^{52}$

$$
\mathcal{H}_{C}^{(\theta \mathbf{x})}=-\frac{3}{4}(3 / \pi)^{1 / 3} e^{2} \int d \overrightarrow{\mathbf{r}}\left[\rho_{p}(\overrightarrow{\mathrm{r}})\right]^{4 / 3} .
$$

TDHF studies of light-ion systems have shown that the inclusion of $\mathcal{H}_{C}^{(\text {ex })}$ has negligible effect on experimental observables. ${ }^{14}$ In the present calculations for ${ }^{84} \mathrm{Kr}+{ }^{209} \mathrm{Bi}$ and ${ }^{84} \mathrm{Kr}{ }^{208} \mathrm{~Pb}, \mathcal{H}_{C}^{(\mathrm{ex})}$ is approximately 30 times smaller than $\mathcal{H}_{C}^{\left(\mathrm{dir}_{\mathrm{r}}\right)}$ and fluctuates little throughout the collision.

\section{Specialization to axial symmetry}

If the nuclear system is assumed to be axially symmetric, it is convenient to express the TDHF equations in cylindrical coordinates $\vec{r}=(r, z, \phi){ }^{2,6}$ The wave functions can then be written in the form

$$
\psi_{\alpha}=\psi_{\alpha}(r, z) e^{i \mu} \alpha^{\Phi},
$$

where $\psi_{\alpha}(r, z)$ depends on the magnitude (but not the sign) of $\mu_{\alpha}$, the azimuthal quantum number.

The energy functional (2.1) can be written as

$$
\mathcal{H}=\mathfrak{H}_{0}+\mathcal{H}_{H}+\mathfrak{H C}_{V} \text {, }
$$

where $\mathfrak{H}_{0}$ contains the derivative-independent parts of $\mathcal{H}_{n}+\mathcal{H}_{C}$, and $\mathcal{H}_{H}$ and $\mathcal{H}_{V}$ contain those terms in $\mathcal{H}_{n}$ arising from $z$ and $r$ derivatives, respectively. ( $\mathcal{H}_{V}$ also contains terms due to the $\phi$ derivative.) In detail,

TABLE I. Parameters of the Skyrme II and modified Skyrme II interactions (Refs. 18, 35, 36).

\begin{tabular}{cccccccccc}
\hline $\begin{array}{c}t_{0} \\
\left.(\mathrm{MeV} \mathrm{fm})^{3}\right)\end{array}$ & $x_{0}$ & $\begin{array}{c}\tilde{t}_{0} \\
\left(\mathrm{MeV} \mathrm{fm}^{3}\right)\end{array}$ & $\tilde{x}_{0}$ & $\begin{array}{c}t_{1} \\
\left(\mathrm{MeV} \mathrm{fm}^{5}\right)\end{array}$ & $\begin{array}{c}t_{2} \\
\left(\mathrm{MeV} \mathrm{fm}^{5}\right)\end{array}$ & $\begin{array}{c}t_{3} \\
\left(\mathrm{MeV} \mathrm{fm}^{6}\right)\end{array}$ & $\begin{array}{c}V_{l} \\
(\mathrm{MeV})\end{array}$ & $\begin{array}{c}V_{u} \\
(\mathrm{MeV})\end{array}$ & $\begin{array}{c}a \\
(\mathrm{fm})\end{array}$ \\
\hline-1169.9 & 0.34 & -104.49 & 4.01 & 585.6 & -27.1 & 9331.0 & -444.85 & -863.53 & 0.45979 \\
\hline \hline
\end{tabular}




$$
\begin{aligned}
\mathcal{H}_{0}=\int d \overrightarrow{\mathrm{r}} & {\left[\frac{1}{2} \tilde{t}_{0}\left(1+\tilde{x}_{0} / 2\right) \rho^{2}-\left(\frac{1}{2}+\tilde{x}_{0}\right) \sum_{q} \rho_{q}{ }^{2}\right.} \\
& \left.+\frac{t_{3}}{8}\left(\rho^{3}-\rho \sum_{q} \rho_{q}{ }^{2}\right)\right]+\mathcal{H}_{y}+\mathcal{H}_{C}, \\
\mathcal{H}_{H}=\int d \overrightarrow{\mathrm{r}} & {\left[\frac{\hbar^{2}}{2 m} \tau_{z}+\frac{t_{1}+t_{2}}{4}\left(\rho \tau_{z}-j_{z}{ }^{2}\right)\right.} \\
& \left.+\frac{\left(t_{2}-t_{1}\right)}{8} \sum_{q}\left(\rho_{q} \tau_{z q}-j_{z q}{ }^{2}\right)\right],
\end{aligned}
$$

and

$$
\begin{aligned}
\mathcal{H}_{V}=\int d \overrightarrow{\mathrm{r}} & {\left[\frac{\hbar^{2}}{2 m}\left(\tau_{r}+s\right)+\frac{t_{1}+t_{2}}{4}\left[\rho\left(\tau_{r}+s\right)-j_{r}{ }^{2}\right]\right.} \\
& \left.+\left(\frac{t_{2}-t_{1}}{8}\right) \sum_{q}\left[\rho_{q}\left(\tau_{r q}+s_{q}\right)-j_{r q}{ }^{2}\right]\right] .
\end{aligned}
$$

For notational convenience, we have introduced the following "components" of the various densities:

$$
\begin{aligned}
& \tau_{z q}=\sum_{\alpha \in q}\left|\partial \psi_{\alpha} / \partial z\right|^{2}, \\
& \tau_{r q}=\sum_{\alpha \in q}\left|\partial \psi_{\alpha} / \partial r\right|^{2}, \\
& s_{\alpha}=\sum_{\alpha \in q}\left|\psi_{\alpha}\right|^{2} \mu_{\alpha}^{2} / r^{2}, \\
& j_{z q}=\sum_{\alpha \in q} \operatorname{Im}\left(\psi_{\alpha}^{*} \partial \psi_{\alpha} / \partial z\right), \\
& j_{r q}=\sum_{\alpha \in q} \operatorname{Im}\left(\psi_{\alpha}^{*} \partial \psi_{\alpha} / \partial r\right) .
\end{aligned}
$$

Note that because we assume equal occupation of pairs of orbitals having equal and opposite azimuthal quantum numbers, $\vec{j}$ has no azimuthal components.

\section{SPATIAL DISCRETIZATION OF THE ENERGY FUNCTIONAL}

We solve the TDHF equations by finite-difference methods on a uniform cylindrical mesh, as discussed in Refs. 6 and 35. This requires a discrete approximation to $\mathfrak{H C}$ in terms of the values of the single-particle wave functions at the mesh points

$$
\begin{aligned}
& r_{i}=\left(i-\frac{1}{2}\right) \Delta r, \\
& z_{j}=(j-1) \Delta z,
\end{aligned}
$$

with $i$ and $j$ integers satisfying $1 \leqslant i \leqslant N_{R},|j|$ $\leqslant N_{z}$, and $\Delta r, \Delta z$ the mesh intervals. Each single- particle wave function is then represented by its values on the mesh points,

$$
\psi_{\alpha}(i, j) \equiv \psi_{\alpha}\left(r_{i}, z_{j}\right),
$$

and is normalized to

$$
\sum_{i, j}\left|\psi_{\alpha}(i, j)\right|^{2} \Delta V_{i}=1
$$

where the volume element is

$$
\Delta V_{i}=2 \pi\left(i-\frac{1}{2}\right)(\Delta r)^{2} \Delta z \text {. }
$$

We also impose the boundary conditions that the $\psi_{\alpha}$ vanish at the mesh edges,

$$
\psi_{\alpha}\left(N_{R}, j\right)=\psi_{\alpha}\left(i, \pm N_{z}\right)=0 .
$$

To discretize $\mathfrak{H}$, we define $\mathrm{e}^{53}$

$$
g_{\alpha}(i, j)=\left(i-\frac{1}{2}\right)^{1 / 2} \psi_{\alpha}(i, j)
$$

and the following discrete approximations to the densities [compare (2.14)]:

$$
\begin{aligned}
& \rho_{q}(i, j)=\frac{1}{\left(i-\frac{1}{2}\right)} \sum_{\alpha \in q}\left|g_{\alpha}(i, j)\right|^{2}, \\
& \rho_{q}\left(i, j+\frac{1}{2}\right)=\frac{1}{2}\left[\rho_{q}(i, j)+\rho_{q}(i, j+1)\right], \\
& \rho_{q}\left(i+\frac{1}{2}, j\right)=\frac{1}{2}\left[\rho_{q}(i, j)+\rho_{q}(i+1, j)\right], \\
& \tau_{z q}\left(i, j+\frac{1}{2}\right)=\frac{1}{\left(i-\frac{1}{2}\right)(\Delta z)^{2}} \sum_{\alpha \in q}\left|g_{\alpha}(i, j)-g_{\alpha}(i, j+1)\right|^{2}, \\
& \tau_{r q}\left(i+\frac{1}{2}, j\right)= \\
& \left(i-\frac{1}{2}\right)(\Delta r)^{2} \\
& \quad \times \sum_{\alpha \in q} r_{i+(1 / 2)}\left|\frac{g_{\alpha}(i, j)}{\sqrt{r_{i}}}-\frac{g_{\alpha}(i+1, j)}{\sqrt{r_{i+1}}}\right|^{2},
\end{aligned}
$$

$$
\begin{aligned}
s_{q}(i, j)=\frac{1}{r_{i}{ }^{2}} & \frac{1}{\left(i-\frac{1}{2}\right)} \sum_{\alpha \in q} \mu_{\alpha}{ }^{2}\left|g_{\alpha}(i, j)\right|^{2} \\
j_{z q}\left(i, j+\frac{1}{2}\right) & =\frac{1}{\left(i-\frac{1}{2}\right) \Delta z} \sum_{\alpha \in q} \operatorname{Im}\left[g_{\alpha}^{*}(i, j) g_{\alpha}(i, j+1)\right], \\
j_{r q}\left(i+\frac{1}{2}, j\right)= & {\left[\frac{i}{\left(i-\frac{1}{2}\right)\left(i^{2}-\frac{1}{4}\right)}\right]^{1 / 2} \frac{1}{\Delta r} } \\
& \times \sum_{\alpha \in q} \operatorname{Im}\left[g_{\alpha}^{*}(i, j) g_{\alpha}(i+1, j)\right]
\end{aligned}
$$

Using the standard lowest-order approximations for the derivatives, and taking care to properly "center" the difference formulas, Eqs. (2.13) may be written as

$$
\begin{aligned}
\mathcal{H}_{0}=\sum_{i, j} \Delta V_{i}\left\{\frac{1}{2} \tilde{t}_{0}\left[\left(1+\tilde{x}_{0} / 2\right) \rho^{2}(i, j)-\left(\frac{1}{2}+\tilde{x}_{0}\right) \sum_{a} \rho_{q}{ }^{2}(i, j)\right]\right. \\
\left.+\frac{t_{3}}{8}\left[\rho^{3}(i, j)-\rho(i, j) \sum_{a} \rho_{a}{ }^{2}(i, j)\right]-\frac{3}{4}\left(\frac{3}{\pi}\right)^{1 / 3} e^{2} \rho_{p}^{4 / 3}(i, j)\right\}+\mathcal{H}_{y}+\mathcal{H}_{C}^{(\text {dir })},
\end{aligned}
$$




$$
\begin{aligned}
\mathcal{F}_{H}=\sum_{i, j} \Delta V_{i}\left\{\frac{\hbar^{2}}{2 m} \tau_{z}\left(i, j+\frac{1}{2}\right)+\frac{t_{1}+t_{2}}{4}\left[\rho\left(i, j+\frac{1}{2}\right) \tau_{z}\left(i, j+\frac{1}{2}\right)-j_{z}{ }^{2}\left(i, j+\frac{1}{2}\right)\right]\right. \\
\left.+\frac{\left(t_{2}-t_{1}\right)}{8}\left[\sum_{q} \rho_{q}\left(i, j+\frac{1}{2}\right) \tau_{z q}\left(i, j+\frac{1}{2}\right)-j_{z q}{ }^{2}\left(i, j+\frac{1}{2}\right)\right]\right\}
\end{aligned}
$$

and

$$
\begin{aligned}
\mathcal{H}_{V}=\sum_{i, j} \Delta V_{i}\{ & \frac{\hbar^{2}}{2 m}\left[\tau_{r}\left(i+\frac{1}{2}, j\right)+s(i, j)\right]+\frac{t_{1}+t_{2}}{4}\left[\rho\left(i+\frac{1}{2}, j\right) \tau_{r}\left(i+\frac{1}{2}, j\right)+\rho(i, j) s(i, j)-j_{r}{ }^{2}\left(i+\frac{1}{2}, j\right)\right] \\
& \left.+\frac{t_{2}-t_{1}}{8}\left[\sum_{a} \rho_{q}\left(i+\frac{1}{2}, j\right) \tau_{r q}\left(i+\frac{1}{2}, j\right)+\rho_{q}(i, j) s_{q}(i, j)-j_{r q}{ }^{2}\left(i+\frac{1}{2}, j\right)\right]\right\}
\end{aligned}
$$

In Eq. (3.8a) $\mathcal{H}_{y}$ and $\mathcal{H}_{C}^{(\mathrm{dir})}$ are the discrete approximations to the finite-range Yukawa and direct Coulomb energies, computed by the methods described in Ref. 6. We note that more sophisticated discretizations of $\mathfrak{H}$ are possible, ${ }^{11}$ although these result in a more complex time evolution. Since our calculations retain only two nontrivial spatial dimensions, storage considerations are not a major problem and we have chosen to use a relatively fine mesh and low-order discretizations.

\section{FINITE DIFFERENCE FORM OF THE TDHF EQUATIONS}

Since the TDHF equations are partial differential equations in space and time, the derivation of difference equations suitable for numerical computation requires two nearly independent steps: a spatial discretization of the equations and a specification of a time-evolution algorithm. We begin by treating the former.

\section{A. Spatial discretization}

One appealing derivation of the TDHF equations starts from a variational principle in which an action functional $g$ is made stationary with respect to variations of the single-particle wave functions $\psi_{\alpha}$ of the trial determinant $\Psi^{50}$ Thus,

$$
\frac{\delta}{\delta \psi_{\alpha}^{*}(\overrightarrow{\mathrm{r}}, t)} g=\frac{\delta}{\delta \psi_{\alpha}^{*}(\overrightarrow{\mathrm{r}}, t)} \int d t\left\langle\Psi\left|i \hbar \frac{\partial}{\partial t}-H\right| \Psi\right\rangle=0,
$$

where $H$ is the many-body Hamiltonian. Our discretization is based on this principle and hence properly embodies those conservation laws (ener- gy and norm) satisfied by the continuous equations. In particular, we discretize (4.1) as

$$
\begin{aligned}
& g=\int d t\left\{\sum_{i j} \Delta V_{i}\left[\sum_{\alpha} \psi_{\alpha}^{*}(i, j) i \hbar \frac{\partial \psi_{\alpha}(i, j)}{\partial t}\right]\right. \\
&\left.-\mathfrak{H}_{0}-\mathfrak{H}_{H}-\mathfrak{H}_{V},\right\}
\end{aligned}
$$

where $\mathfrak{H}_{0}, \mathfrak{H}_{H}$, and $\mathfrak{H}_{V}$ are the discretizations giv en by (3.8). Variation of (4.2) with respect to $\psi_{\alpha}^{*}(i, j)$ [or, equivalently, $\left.g_{\alpha}^{*}(i, j)\right]$ then results in TDHF equations of the form

$$
i \hbar \frac{\partial g_{\alpha}(i, j)}{\partial t}=\left(H g_{\alpha}\right)(i, j)+\left(V g_{\alpha}\right)(i, j) \text {. }
$$

The action of the "horizontal" Hamiltonian $H$ is defined by

$$
\begin{aligned}
\left(H g_{\alpha}\right)(i, j)= & B_{\alpha}^{(+)}(i, j) g_{\alpha}(i, j+1) \\
& +B_{\alpha}^{(+) *}(i, j-1) g_{\alpha}(i, j-1) \\
& +\left[B_{\alpha}^{(0)}(i, j)+\frac{1}{2} h_{0_{q}}(i, j)\right] g_{\alpha}(i, j),
\end{aligned}
$$

and the "vertical" Hamiltonian, $V$ by

$$
\begin{aligned}
\left(V g_{\alpha}\right)(i, j)= & A_{\alpha}^{(+)}(i, j) g_{\alpha}(i+1, j) \\
& +A_{q}^{(+) *}(i-1, j) g_{\alpha}(i-1, j) \\
& +\left[A_{\mu_{\alpha}}^{(0)}(i, j)+\frac{1}{2} h_{0 q}(i, j)\right] g_{\alpha}(i, j) .
\end{aligned}
$$

In these expressions, $\mu$ and $q$ are the azimuthal and isospin quantum numbers of the orbital $\alpha$. Note that (4.4) and (4.5) show that if $B^{(0)}, A^{(0)}$, and $h_{0}$ are real (as we show below), then $H$ and $V$ are Hermitian operators, so that (4.3) implies a unitary evolution of $g$.

The quantity $h_{0_{q}}(i, j)$ results from the variation of $\mathcal{H}_{0}$ and is given by

$$
\begin{aligned}
h_{0 q}(i, j)= & \tilde{t}_{0}\left[\left(1+\tilde{x}_{0} / 2\right) \rho(i, j)-\left(\frac{1}{2}+\tilde{x}_{0}\right) \rho_{q}(i, j)\right]+\frac{t_{3}}{4}\left[\rho^{2}(i, j)-\rho_{q}^{2}(i, j)\right]+V_{u} U_{y}(i, j) \\
& +\left(V_{l}-V_{u}\right) U_{y q}(i, j)+\delta_{\alpha p}\left[U_{C}(i, j)-\left(\frac{3}{\pi}\right)^{1 / 3} e^{2} \rho_{p}^{1 / 3}(i, j)\right]
\end{aligned}
$$

where $U_{y a}(i, j)$ and $U_{C}(i, j)$ are the discrete approximations to the Yukawa and direct Coulomb potentials 


$$
\begin{aligned}
& U_{y q}(\overrightarrow{\mathbf{r}})=\int d \overrightarrow{\mathbf{r}}^{\prime} \frac{e^{-\left|\overrightarrow{\mathbf{r}}^{\prime} \overrightarrow{\mathbf{r}}^{\prime}\right| / a}}{\left|\overrightarrow{\mathbf{r}}-\overrightarrow{\mathbf{r}}^{\prime}\right| / a} \rho_{q}\left(\overrightarrow{\mathbf{r}}^{\prime}\right), \\
& U_{c}(\overrightarrow{\mathrm{r}})=e^{2} \int d \overrightarrow{\mathbf{r}}^{\prime} \frac{1}{\left|\overrightarrow{\mathbf{r}}-\overrightarrow{\mathbf{r}}^{\prime}\right|} \rho_{p}\left(\overrightarrow{\mathbf{r}}^{\prime}\right) .
\end{aligned}
$$

These are evaluated by solving, respectively, the discrete Helmholtz and Poisson equations using Gaussian elimination. ${ }^{6,54}$

The quantities $B_{q}$ arise from the variation of $\mathcal{H}_{H}$ :

$$
\begin{aligned}
B_{a}^{(0)}(i, j)= & \frac{\hbar^{2}}{m(\Delta z)^{2}}+\frac{t_{1}+t_{2}}{4}\left\{\frac{\rho\left(i, j+\frac{1}{2}\right)+\rho\left(i, j-\frac{1}{2}\right)}{(\Delta z)^{2}}+\frac{1}{2}\left[\tau\left(i, j+\frac{1}{2}\right)+\tau\left(i, j-\frac{1}{2}\right)\right]\right\} \\
& +\frac{\left(t_{2}-t_{1}\right)}{8}\left\{\frac{\rho_{o}\left(i, j+\frac{1}{2}\right)+\rho_{q}\left(i, j-\frac{1}{2}\right)}{(\Delta z)^{2}}+\frac{1}{2}\left[\tau_{q}\left(i, j+\frac{1}{2}\right)+\tau_{q}\left(i, j-\frac{1}{2}\right)\right]\right\} ;
\end{aligned}
$$

and

$$
\begin{aligned}
B_{q}^{(+)}(i, j)= & -\frac{\hbar^{2}}{2 m(\Delta z)^{2}}-\frac{\left(t_{1}+t_{2}\right)}{4(\Delta z)^{2}}\left[\rho\left(i, j+\frac{1}{2}\right)+\frac{\Delta z}{\sqrt{-1}} j_{z}\left(i, j+\frac{1}{2}\right)\right] \\
& -\frac{\left(t_{2}-t_{1}\right)}{8(\Delta z)^{2}}\left[\rho_{q}\left(i, j+\frac{1}{2}\right)+\frac{\Delta z}{\sqrt{-1}} j_{z q}\left(i, j+\frac{1}{2}\right)\right],
\end{aligned}
$$

where, in order to avoid confusion, we have written the unit imaginary explicitly as $\sqrt{-1}$. Similarly, the $A_{\mu_{q}}$ are obtained from the variation of $\mathcal{H}_{V}$ :

$$
\begin{aligned}
A_{\mu q}^{(0)}(i, j)= & C_{\mu_{q}}(i, j)+D_{q}(i, j) ; \\
A_{q}^{(+)}(i, j)= & \frac{i}{\left(i^{2}-\frac{1}{4}\right)^{1 / 2}}\left[-\frac{\hbar^{2}}{2 m(\Delta r)^{2}}-\frac{\left(t_{1}+t_{2}\right)}{4(\Delta r)^{2}} \rho\left(i+\frac{1}{2}, j\right)-\frac{\left(t_{2}-t_{1}\right)}{8(\Delta r)^{2}} \rho_{q}\left(i+\frac{1}{2}, j\right)\right] \\
& -\frac{\Delta r}{\sqrt{-1}}\left(\frac{i}{i-\frac{1}{2}}\right)^{1 / 2}\left[\frac{\left(t_{1}+t_{2}\right)}{4(\Delta r)^{2}} j_{r}\left(i+\frac{1}{2}, j\right)+\frac{\left(t_{2}-t_{1}\right)}{8(\Delta r)^{2}} j_{r q}\left(i+\frac{1}{2}, j\right)\right] ; \\
C_{\mu q}(i, j)= & \frac{\mu^{2}}{r_{i}^{2}}\left[\frac{\hbar^{2}}{2 m}+\left(\frac{t_{1}+t_{2}}{4}\right) \rho(i, j)+\left(\frac{t_{2}-t_{1}}{8}\right) \rho_{q}(i, j)\right] ;
\end{aligned}
$$

and

$$
\begin{aligned}
D_{a}(i, j)= & \frac{\hbar^{2}}{m(\Delta r)^{2}}+\frac{t_{1}+t_{2}}{4} s(i, j)+\frac{\left(t_{2}-t_{1}\right)}{8} s_{q}(i, j) \\
& +\frac{\left(t_{1}+t_{2}\right)}{4}\left[\left(\frac{i}{i-\frac{1}{2}} \rho\left(i+\frac{1}{2}, j\right)+\frac{i-1}{i-\frac{1}{2}} \rho\left(i-\frac{1}{2}, j\right)\right) /(\Delta r)^{2}+\frac{1}{2}\left(\tau_{r}\left(i+\frac{1}{2}, j\right)+\frac{i-\frac{3}{2}}{i-\frac{1}{2}} \tau_{r}\left(i-\frac{1}{2}, j\right)\right)\right] \\
& +\frac{t_{2}-t_{1}}{8}\left[\left(\frac{i}{i-\frac{1}{2}} \rho_{q}\left(i+\frac{1}{2}, j\right)+\frac{i-1}{i-\frac{1}{2}} \rho_{q}\left(i-\frac{1}{2}, j\right)\right) /(\Delta r)^{2}+\frac{1}{2}\left(\tau_{r q}\left(i+\frac{1}{2}, j\right)+\frac{i-\frac{3}{2}}{i-\frac{1}{2}} \tau_{r q}\left(i-\frac{1}{2}, j\right)\right)\right]
\end{aligned}
$$

Note that the quantities $h_{0 q}, B_{q}^{(0)}, B_{q}^{(+)}, D_{q}$, and $A_{q}^{(+)}$are independent of $\mu$ and thus may be generated once for all $\mu$. In contrast, $C_{\mu_{q}}$ and $A_{\mu_{q}}^{(0)}$ depend upon $\mu$ as well and must be generated separately for each azimuthal quantum number of a given isospin type. Thus, the various quantities need not be generated each time (4.3) is solved for a given $\alpha$, but may be calculated for blocks of states grouped by $\mu$ and $q$, resulting in a substantial increase in computational speed.

\section{B. Time discretization}

In this subsection, we suppress all labels indicating quantum numbers and the spatial charac- terization and rewrite (4.3) as

$$
i \hbar \frac{\partial g(t)}{\partial t}=(H+V) g(t) \equiv h(t) g(t)
$$

where $h$ is an Hermitian operator. This equation is formally solved by

$$
g(t)=U\left(t, t_{0}\right) g\left(t_{0}\right),
$$

where the unitary time evolution operator is

$$
U\left(t, t_{0}\right)=T \exp \left[-i / \hbar \int_{t_{0}}^{t} h(\tau) d \tau\right]
$$

and $T$ is the Dyson time-ordering operator.

To discretize in time, we define the mesh 


$$
t_{n}=n \Delta t, \quad n=0,1,2, \ldots
$$

and write (4.12) in the recursive form

$$
g^{(n+1)}=U(n+1, n) g^{(n)},
$$

with $U(n+1, n)=U\left(t_{n+1}, t_{n}\right)$, and $g^{(n)}=g\left(t_{n}\right)$. If we temporarily assume $h$ to be time independent over the interval $\left[t_{n}, t_{n+1}\right]$ (see the next subsection), Eq. (4.13) becomes

$$
\begin{aligned}
U(n+1, n) & =e^{-i h \Delta t / \hbar} \\
& =1-\frac{i \Delta t}{\hbar} h+\frac{1}{2}\left(-\frac{i \Delta t}{\hbar} h\right)^{2}+\cdots
\end{aligned}
$$

An explicit algorithm for solving (4.14) is to truncate the expansion in Eq. (4.15b) to represent the exact exponential operator (4.15a) as accurately as desired. In our two-dimensional calculations in cylindrical coordinates, this method leads to numerical instabilities which we believe are associated with the coordinate singularity at $r=0$. However, the method seems to be stable in Cartesian coordinates and is being successfully used by several groups (Refs. 11-13, 15, 23, 29, $33,34)$.

An implicit stable algorithm for (4.14) is the Crank-Nicholson (CN) operator ${ }^{1}$

$$
U \approx U_{\mathrm{CN}}=\frac{1-\frac{i}{2 \hbar} \Delta t h}{1+\frac{i}{2 \hbar} \Delta t h},
$$

which is the $[1,1]$ Padé approximation to the exponential. This operator is exactly unitary and approximates (4.15) through $\theta(\Delta t)^{2}$. However, since $h$ is an Hermitian $2 N_{R} \cdot N_{Z} \times 2 N_{R} \cdot N_{Z}$ band matrix of half bandwidth $N_{R}+1$, the inversion in (4.16) is difficult to perform, and it is useful to consider two other approximations.

The first of these is the Peaceman-Rachford (PR) method, ${ }^{6,55}$ in which

$$
\begin{aligned}
U \approx U_{\mathrm{PR}}= & {\left[\frac{1}{1+\left(\frac{i \Delta t}{2 \hbar}\right) V}\right]\left[\frac{1-\left(\frac{i \Delta t}{2 \hbar}\right) H}{1+\left(\frac{i \Delta t}{2 \hbar}\right) H}\right] } \\
& \times\left[1-\left(\frac{i \Delta t}{2 \hbar}\right) V\right] .
\end{aligned}
$$

This expression approximates the exponential time evolution operator in Eq. (4.15) through $\mathcal{O}(\Delta t)^{2}$ even if $H$ and $V$ do not commute. The operator is not unitary, but by successive applications over several time steps it results in almost unitary evolution. Since $H$ and $V$ are represented by tridiagonal matrices [cf. (4.4) and (4.5)], the inversions appearing in Eq. (4.17) may be performed very rapidly by Gaussian elimination. ${ }^{54}$

To solve (4.14), we evaluate in succession

$$
\begin{aligned}
& g_{1}^{(n)}=\left[1-\left(\frac{i \Delta t}{2 \hbar}\right) V\right] g^{(n)}, \\
& g_{2}^{(n)}=\left[1+\left(\frac{i \Delta t}{2 \hbar}\right) H\right]^{-1} g_{1}^{(n)}, \\
& g_{3}^{(n)}=\left[1-\left(\frac{i \Delta t}{2 \hbar}\right) H\right] g_{2}^{(n)},
\end{aligned}
$$

and finally

$$
g^{(n+1)}=\left[1+\left(\frac{i \Delta t}{2 \hbar}\right) V\right]^{-1} g_{3}^{(n)},
$$

so that two explicit operations and two inversions are required. However, we note that $t^{56}$

$$
\begin{aligned}
g_{3}^{(n)} & =\left\{2-\left[1+\left(\frac{i \Delta t}{2 \hbar}\right) H\right]\right\} g_{2}^{(n)} \\
& =2 g_{2}^{(n)}-g_{1}^{(n)},
\end{aligned}
$$

which speeds up the calculation by eliminating the explicit operation (4.18c).

An alternative approximation to (4.16) is the local one-dimensional (LOD) method, ${ }^{57}$ in which

$$
U \approx\left[\frac{1-\left(\frac{i \Delta t}{2 \hbar}\right) V}{1+\left(\frac{i \Delta t}{2 \hbar}\right) V}\right]\left[\frac{1-\left(\frac{i \Delta t}{2 \hbar}\right) H}{1+\left(\frac{i \Delta t}{2 \hbar}\right) H}\right],
$$

or

$$
U \approx\left[\frac{1-\left(\frac{i \Delta t}{2 \hbar}\right) H}{1+\left(\frac{i \Delta t}{2 \hbar}\right) H}\right]\left[\frac{1-\left(\frac{i \Delta t}{2 \hbar}\right) V}{1+\left(\frac{i \Delta t}{2 \hbar}\right) V}\right] .
$$

The LOD operators are exactly unitary but, unlike the PR operator, do not approximate the exponential operator through $O(\Delta t)^{2}$ unless $H$ and $V$ commute. To solve Eq. (4.14), equations similar to (4.19) can be used to eliminate all explicit operations. The LOD method is thus about 10-15\% faster than PR. However, the latter appears to be more stable for a given $\Delta t$. For example, in TDHF studies of ${ }^{16} \mathrm{O}+{ }^{40} \mathrm{Ca}$ using $\Delta t=0.0025 \times 10^{-21}$ $\mathrm{s}$, the total energy is conserved to within $0.6 \mathrm{MeV}$ for PR but varies by as much as $3.7 \mathrm{MeV}$ for LOD. All calculations reported here have been done with the PR method.

\section{Methods for constructing $h$}

We now consider the construction of the HF Hamiltonian $h$ effecting a time step from $t_{n}$ to $t_{n+1}=t_{n}$ $+\Delta t$ according to the methods given in the previous section. From Eq. (4.13), it is clear that $h$ approximates the Hamiltonian between time $t_{n}$ and $t_{n+1}$. Since the wave functions at $t_{n+1}$ are not yet known at $t_{n}$ (indeed, they depend upon $h$ ), $h$ is specified only in some implicit manner. As the 
simplest approximation $\left(h=h^{(n)}\right.$, where $h^{(n)}$ is constructed from the wave function at $t_{n}$ ) is known to lead to serious violation of energy conservation, ${ }^{1}$ we discuss two different prescriptions for generating $h$.

The first prescription is essentially the double time stepping (Hamiltonian averaging) method described in Appendix B of Ref. 1. We first construct

$$
\bar{g}=U^{(n)} g^{(n)},
$$

with $U^{(n)}$ defined by $h^{(n)}$ and either (4.17) or

(4.20). The average densities,

$$
f=\frac{1}{2}\left(f^{(n)}+\bar{f}\right),
$$

where $f=\rho, \tau, \overrightarrow{\mathrm{j}}$, or $s$ defined by Eqs. (3.7) are then used to define $h$, allowing (4.14) to be solved for $g^{(n+1)}$.

In the second prescription, ${ }^{1} \bar{g}$ is calculated from Eq. (4.21) with $\Delta t$ replaced by $\Delta t / 2$, and the resulting densities $\bar{\rho}, \bar{\tau}, \bar{j}$, and $\bar{s}$ used to define $h$. As in the first method, Eq. (4.14) is then solved to determine $g^{(n+1)}$

Both of these methods involve solving the TDHF equations twice for each time step. We find that the second method allows the use of somewhat longer time steps than the first method, as predicted in Ref. 1. Other ways of extrapolating the densities to $t_{n}+\frac{1}{2} \Delta t$ were also investigated, such as utilizing the equation of continuity or polynomial extrapolation from previous time steps. These were not as stable as either of the two prescriptions discussed above.

\section{THE ROTATING FRAME APPROXIMATION}

Our calculations assume an axial symmetry about the line joining the mass centers of the colliding ions. In order to simulate nonzero impactparameter collisions, we follow Refs. 2, 6, 7, and 13 and assume that the symmetry axis rotates in space and perform our calculations in the body -fixed frame. The details of how this picture is implemented are discussed in the references cited. We only outline the method here.

We add to the energy functional (2.1) the classical rotational energy

$$
\mathfrak{H}_{R}=L^{2} / 2 I[\rho],
$$

where

$$
L \equiv\left(l+\frac{1}{2}\right) \hbar
$$

is the conserved total angular momentum along the rotation axis normal to the reaction plane, and the moment of inertia $I[\rho]$ is a functional of the density. The single-particle potentials $h_{0 q}$ appearing in Eq. (4.6) must then be redefined as

$$
h_{0 q}-h_{0 q}-\frac{1}{2} \omega^{2} \frac{\delta I[\rho]}{\delta \rho(\overrightarrow{\mathrm{r}})},
$$

where

$$
\omega=\frac{d \theta}{d t}=L / I[\rho]
$$

is the rate of rotation of the symmetry axis. Coriolis forces are completely neglected in this approximation.

At the beginning of the collision, when the ions are far apart, the moment of inertia is assumed to be that of two point masses

$$
I[\rho]=\mu R^{2}, \quad \rho_{\min }(t)<\rho_{c},
$$

where $\mu$ is the ion-ion reduced mass and

$$
\begin{aligned}
R= & \frac{2 \pi}{A_{L}} \int_{-\infty}^{z_{\min }} d z\left|z-z_{\min }\right| \int_{0}^{\infty} r d r \rho(r, z) \\
& +\frac{2 \pi}{A_{R}} \int_{x_{\min }}^{\infty} d z\left|z-z_{\min }\right| \int_{0}^{\infty} r d r \rho(r, z)
\end{aligned}
$$

is the separation distance between the two ions. In this expression, $z_{\min }$ is the location of the minimum density $\rho_{\mathrm{min}}$ along the symmetry axis between the two ions, and is used to divide the system into two parts (left and right) of masses $A_{L}$ and $A_{R}$

$$
\begin{aligned}
& A_{L}=2 \pi \int_{-\infty}^{x_{\min }} d z \int_{0}^{\infty} r d r \rho(r, z), \\
& A_{R}=A-A_{L}=\int_{z_{\min }}^{\infty} d z \int_{0}^{\infty} r d r \rho(r, z) .
\end{aligned}
$$

Note that the dividing plane $z=z_{\min }$ is defined unambiguously only when the two ions are well separated (at the beginning or end of a collision). The constant $\rho_{c}$ in Eq. (5.5) is the "clutching" density, which we take to be equal to $\frac{1}{2} \rho_{0}=0.0725 \mathrm{fm}^{-3}$, one-half the saturation density of nuclear matter. When $\rho_{\min }(t)$ exceeds $\rho_{c}$, the nuclei are assumed to have clutched and the moment of intertia is taken to be that of a rigid body

$$
\begin{aligned}
& I[\rho]=2 \pi m \int_{-\infty}^{\infty} d z \int_{0}^{\infty} r d r \rho(r, z)\left(z^{2}+\frac{1}{2} r^{2}\right), \\
& \rho_{\min }(t) \geqslant \rho_{C},
\end{aligned}
$$

and we work in a coordinate system where the overall center of mass is at $z=0$.

The prescription outlined above corresponds to method $R 2$ of Ref. 13, where it and a number of other rotating frame approximations were compared in detail with results from fully three-dimensional calculations. Of particular significance was a study of ${ }^{40} \mathrm{Ca}+{ }^{40} \mathrm{Ca}$, in which the relative velocity above the Coulomb barrier was chosen to 
be approximately equal to that of ${ }^{84} \mathrm{Kr}+{ }^{209} \mathrm{Bi}$ at $E_{1 \mathrm{ab}}=600 \mathrm{MeV}$, one of the systems studied in this paper. In this case, the method we use was found to give the best overall reproduction of the threedimensional results. ${ }^{13}$ It should also be mentioned that some calculations have demonstrated that our results for ${ }^{84} \mathrm{Kr}+{ }^{209} \mathrm{Bi}$ at $E_{1 \mathrm{ab}}=600 \mathrm{MeV}$ are insensitive to increases in $\rho_{C}$ from $0.0725 \mathrm{fm}^{-3}$ to $0.1100 \mathrm{fm}^{-3}$.

\section{OTHER CALCULATIONAL DETAILS}

\section{A. The filling approximation}

For TDHF calculations involving a deformed target or projectile, there is a substantial ambiguity involving the initial orientation of the ions. The correct way to resolve this would be to perform a suitable average over all such orientations. However, because such orientation effects are expected to be small in the violent collisions we consider, and because such averaging would involve substantially more computing effort, we have employed a filling approximation which uniformly distributes valence nucleons over unfilled shells in the target or projectile and results in spherical ions before the collision. Indeed, this may be viewed as a kind of crude orientation averaging. We therefore generalize Eqs. (2.3) to read

$$
\rho_{\alpha}(\overrightarrow{\mathrm{r}}, t)=\sum_{\alpha \in q} n_{\alpha}\left|\psi_{\alpha}(\overrightarrow{\mathrm{r}}, t)\right|^{2},
$$

and similarly for $\tau$ and $\vec{j}$. The time-independent occupation factors $n_{\alpha}$ are taken to be unity for the filled shells and fractional values for the occupied levels of the unfilled shells. It should be emphasized that since the $n_{\alpha}$ are not all zero or unity, the many-body wave function cannot be expressed as a single Slater determinant, and therefore we do not perform true Hartree-Fock or TDHF calculations. However, once we define the single-particle densities by Eq. (6.1), the entire TDHF evolution scheme is still applicable, and we regard the filling approximation as only a slight generalization of the ordinary theory.

Table II summarizes the filling approximation as applied to the static Hartree-Fock calculations of the initial spherical nuclei needed for our calculations. These occupation factors remain time independent in the dynamical calculation.

\section{B. The initial conditions}

The wave function at time $t=0$ must be chosen to represent an impending collision between the target and projectile, each in its ground state. To generate this wave function, we perform static
TABLE II. Spherical filling approximation for ${ }^{84} \mathrm{Kr}$, ${ }^{208} \mathrm{~Pb}$, and ${ }^{209} \mathrm{Bi}$. The occupation factors $n_{\alpha}$ are unity for all of the orbitals of the filled spherical shells.

\begin{tabular}{|c|c|c|c|}
\hline Nucleus & Isospin & $\begin{array}{l}\text { Last unfilled } \\
\text { spherical } \\
\text { shell }\end{array}$ & $\begin{array}{c}n_{\alpha} \text { for orbitals in the } \\
\text { last shell }\end{array}$ \\
\hline \multirow{4}{*}{${ }^{84} \mathrm{Kr}$} & protons & $1 p-0 f$ & 1 for the $0 f$ orbitals \\
\hline & & & $\frac{1}{3}$ for the $1 p$ orbitals \\
\hline & neutrons & $2 s-1 d-0 g$ & $\frac{4}{9}$ for the $0 g$ orbitals \\
\hline & & & 0 for all others \\
\hline \multirow[t]{4}{*}{${ }^{208} \mathrm{~Pb}$} & protons & $2 p-1 f-0 h$ & $\frac{6}{11}$ for the $0 h$ orbitals \\
\hline & & & 0 for all others \\
\hline & neutrons & $3 s-2 d-1 g-0 i$ & $\frac{7}{13}$ for the $0 i$ orbitals \\
\hline & & & 0 for all others \\
\hline \multirow{4}{*}{${ }^{209} \mathrm{Bi}$} & & & $\frac{13}{22}$ for the $0 h$ orbitals \\
\hline & protons & $2 p-1 f-0 h$ & 0 for all others \\
\hline & neutrons & $3 s-2 d-1 g-0 i$ & $\frac{7}{13}$ for the $0 i$ orbitals \\
\hline & & & 0 for all others \\
\hline
\end{tabular}

HF calculations for each ion with the same energy functional used in the dynamical calculations. This is essential to avoid unphysical oscillations of the nuclei before they collide. The static HF equations for each nucleus have been solved by a coordinate-space method using the Lanczos algorithm. ${ }^{35}$ An alternative which should be employed in future calculations is the imaginary time technique, ${ }^{58}$ which is roughly a factor of 50 faster than the Lanczos method. In Table III we list the rms charge and mass radii and binding energies per nucleon of our static solutions for ${ }^{84} \mathrm{Kr},{ }^{208} \mathrm{~Pb}$, and ${ }^{209} \mathrm{Bi}$.

To construct the TDHF initial conditions, we place the projectile static solution on the left-hand side $(z<0)$ of the mesh and the target static solution on the right-hand side $(z>0)$, so that the over-

TABLE III. Calculated binding energies and $\mathrm{rms}$ radii for ${ }^{84} \mathrm{Kr},{ }^{208} \mathrm{~Pb}$, and ${ }^{209} \mathrm{Bi}$. The charge radius $\boldsymbol{r}_{c}$ is obtained from $r_{c}{ }^{2}=r_{p}{ }^{2}+0.64 \mathrm{fm}^{2}$, where $r_{p}$ is the calculated point proton rms radius. Experimental values for radii (Ref. 59), when known, and binding energies (Ref. 60) are given in parentheses.

\begin{tabular}{|c|c|c|c|}
\hline Nucleus & $\begin{array}{l}\text { rms charge } \\
\text { radius, } r_{c}(\mathrm{fm})\end{array}$ & $\begin{array}{l}\text { rms mass } \\
\text { radius, } r_{m}(\mathrm{fm})\end{array}$ & $\begin{array}{c}\text { Binding } \\
\text { energy per } \\
\text { nucleon } \\
(\mathrm{MeV})\end{array}$ \\
\hline${ }^{84} \mathrm{Kr}$ & 4.18 & 4.17 & $8.77(8.72)$ \\
\hline${ }^{208} \mathrm{~Pb}$ & $5.53(5.50)$ & 5.57 & $7.86(7.87)$ \\
\hline${ }^{209} \mathrm{Bi}$ & $5.55(5.51)$ & 5.58 & $7.87(7.85)$ \\
\hline
\end{tabular}


all center of mass is at $z=0$ and the fragments are separated by $R_{\mathrm{in}}=19 \mathrm{fm}$. The presumably small effects of Coulomb-induced polarization before this time are therefore neglected. The rotating frame orientation $\theta_{\text {in }}$ and relative radial velocity $\dot{R}_{\text {in }}$ are obtained by matching asymptotically to Rutherford trajectories ${ }^{61}$

$$
\begin{aligned}
\theta_{i n}= & \pi-\cos ^{-1}\left(1 / \epsilon_{i n}\right) \\
& +\cos ^{-1}\left[\epsilon_{i n}^{-1}\left(1+\frac{L^{2}}{\mu Z_{L} Z_{R} e^{2} R_{i n}}\right)\right],
\end{aligned}
$$

and

$$
\dot{R}_{\mathrm{in}}=\left(\frac{2}{\mu}\right)^{1 / 2}\left[E_{\text {c. m. }}-\frac{Z_{L} Z_{R} e^{2}}{R_{\mathrm{in}}}-\frac{L^{2}}{2 \mu R_{\mathrm{in}}^{2}}\right]
$$

where

$$
\epsilon_{\mathrm{in}}=\left\{1+\left[\frac{2 E_{\mathrm{c} . \mathrm{m} .} L^{2}}{\mu\left(Z_{L} Z_{R} e^{2}\right)^{2}}\right]\right\}^{1 / 2},
$$

and $Z_{L}$ and $Z_{R}$ are the projectile and target charges. The initial center of mass and laboratory energies are related by

$$
E_{\text {c.m. }}=\frac{A_{R}}{A_{L}+A_{R}} E_{1 \mathbf{2 b}},
$$

with $A_{L}$ and $A_{R}$ the projectile and target masses, while the initial relative momentum $p=\hbar k$ is given by

$$
\hbar k=\mu \dot{R}_{\mathrm{in}} \text {. }
$$

In the center-of-mass system, the projectile and target then move with velocities

$$
v_{L}=\hbar k /\left(A_{L} m\right) \text {, }
$$

and

$$
v_{R}=-\hbar k /\left(A_{R} m\right),
$$

along the symmetry axis.

To induce the initial relative motion, the projectile static solutions are multiplied by the planewave phase $\exp \left(i k z / A_{L}\right)$. Similarly, the target wave functions are boosted by $\exp \left(-i k z / A_{R}\right)$. In the absence of an ion-ion interaction, these solutions would be expected to translate uniformly at the proper velocity. Unfortunately, owing to our spatial discretization, there is an inherent unavoidable error in the initial center-of-mass energy. ${ }^{18,48}$ In a single Cartesian coordinate, the three-point approximation to the kinetic energy is

$$
\begin{aligned}
-\frac{\hbar^{2}}{2 m}\left(D_{z}^{2} \psi\right)(j) & =-\frac{\hbar^{2}}{2 m(\Delta z)^{2}}[\psi(j+1)-2 \psi(j)+\psi(j-1)] \\
& =-\frac{\hbar^{2}}{2 m}\left[\frac{d^{2} \psi}{d z^{2}}+\frac{(\Delta z)^{2}}{12} \frac{d^{4} \psi}{d z^{4}}+\cdots\right] .(6.8)
\end{aligned}
$$

The resulting error of $\theta(\Delta z)^{2}$ in $\left\langle\psi\left|\left(-\hbar^{2} / 2 m\right) D_{z}{ }^{2}\right| \psi\right\rangle$ can be shown to be negative definite and so the calculated mesh kinetic energy is slightly smaller than the analytical value. We have therefore used an iterative procedure to slightly readjust $k$ at $t$ $=0$ in order to guarantee the correct numerically calculated energy. For mesh spacings $\Delta z \leqslant 0.6$ $\mathrm{fm}$, there is convergence in two or three iterations. For additional discussion of spatial discretization errors, see Ref. 18.

\section{Calculation of final state quantities}

At some time after a collision has occurred and the fragments are separating, we employ the following formulas to calculate several quantities describing the final state.

The asymptotic center-of-mass scattering angle and the total fragment kinetic energy are obtained by matching to a pure Rutherford trajectory ${ }^{61}$ :

$$
\begin{aligned}
\theta_{s} & =\theta_{f}-\cos ^{-1}\left(1 / \epsilon_{f}\right) \\
& +\cos ^{-1}\left[\frac{1}{\epsilon_{f}}\left(1+\frac{L_{f}^{2}}{\mu_{f} Z_{f L} Z_{f R} e^{2} R_{f}}\right)\right]
\end{aligned}
$$

and

$$
E_{f}=\frac{\mu_{f}}{2} \dot{R}_{f}^{2}+\frac{L_{f}^{2}}{2 \mu R_{f}^{2}}+\frac{Z_{f L} Z_{f R} e^{2}}{R_{f}} .
$$

Here, $\theta_{f}, \dot{\theta}_{f}, R_{f}$, and $\dot{R}_{f}$ are the frame orientation, angular velocity, fragment separation, and fragment separation velocity at the time of matching; $L_{f}=\mu_{f} R_{f}{ }^{2} \dot{\theta}_{f}$ in the final orbital angular momentum as given by our rotating frame prescription,

$$
\epsilon_{f}=\left\{1+\left[\frac{2 E_{f} L_{f}^{2}}{\mu_{f}\left(Z_{f L} Z_{f R} e^{2}\right)^{2}}\right]\right\}^{1 / 2},
$$

and $\mu_{f}=A_{f L} A_{f R} / A$ is the final ion-ion reduced mass. The final fragment masses and charges are calculated from formulas similar to (5.7), while $R_{f}$ is calculated from (5.6). The fragment separation velocity $\dot{R}_{f}$ may be calculated from $R_{f}$ by the following simple procedure. Knowing $R^{(n)}$ $=R_{f}$ and $R^{(n-1)}$, the values of $R$ at $t_{n}$ and $t_{n-1}$, we may construct

$$
\dot{R}^{(n-1 / 2)}=\left[R^{(n)}-R^{(n-1)}\right] / \Delta t,
$$

and realize that

$$
\begin{aligned}
\dot{R}_{f}=\dot{R}^{(n)} & \approx \dot{R}^{[n-(1 / 2)]}+\frac{1}{2} \Delta t \ddot{R}^{[n-(1 / 2)]} \\
& \approx \dot{R}^{[n+(1 / 2)]}+\frac{1}{2} \Delta t \ddot{R}^{(n)} .
\end{aligned}
$$

However, from the Rutherford trajectory, we may compute

$$
\ddot{R}^{(n)}=\frac{1}{\mu_{f}}\left[\frac{L_{f}^{2}}{\mu_{f}\left(R^{(n)}\right)^{3}}+\frac{Z_{f L} Z_{f R} e^{2}}{\left(R^{(n)}\right)^{2}}\right] .
$$

Equations (6.13b), (6.12), and (6.14) may then be used to estimate $\dot{R}^{(n)}=\dot{R}_{f}$, and hence $E_{f}$ from 
(6.10).

It is also of interest to compute the dispersions in the final fragment charge and mass distributions, in addition to their mean values, $Z_{f L}$ and $A_{f L}$. For a single determinant, a general formula for the dispersion of the number distribution of a given isospin type $q(=p$ or $n)$ in the left-hand fragment is ${ }^{1}$

$$
\sigma_{q}{ }^{2}=\operatorname{Tr}\left(\rho_{q}^{(L)}-\left[\rho_{q}^{(L)}\right]^{2}\right),
$$

where the trace is with respect to all single-particle coordinates, and the "left-hand" density matrix is

$$
\begin{aligned}
\rho_{\alpha}^{(L)}\left(\overrightarrow{\mathbf{r}}, \overrightarrow{\mathbf{r}}^{\prime}\right)= & \theta\left(z_{\min }-z\right)\left[\sum_{\alpha \in Q} n_{\alpha} \psi_{\alpha}(\overrightarrow{\mathbf{r}}) \psi_{\alpha}^{*}\left(\overrightarrow{\mathbf{r}}^{\prime}\right)\right] \\
& \times \theta\left(z_{\min }-z^{\prime}\right) .
\end{aligned}
$$

Here,

$$
\begin{aligned}
\theta(x) & =1, \text { for } x \geqslant 0 \\
& =0, \text { for } x<0
\end{aligned}
$$

is the unit step function and $n_{\alpha}$ is unity for all occupied single-particle orbitals in the determinant. From (6.15) and (6.16), it is easy to show that

$$
\sigma_{q}^{2}=\sum_{\alpha \in q} n_{\alpha} \omega_{\alpha \alpha}-\sum_{\alpha, \beta \in q} n_{\alpha} n_{\beta}\left|\omega_{\alpha \beta}\right|^{2},
$$

where the "left-hand" overlap integrals are

$$
\omega_{\alpha \beta}=2 \pi \int_{-\infty}^{z_{\min }} d z \int_{0}^{\infty} r d r \psi_{\alpha}^{*}(\overrightarrow{\mathrm{r}}) \psi_{\beta}(\overrightarrow{\mathrm{r}}) .
$$

For a Gaussian isospin distribution, the full width at half maximum is

$$
\Gamma_{q}=(8 \ln 2)^{1 / 2} \sigma_{q},
$$

and the full width of the mass distribution is

$$
\Gamma_{A}=\left(\Gamma_{p}^{2}+\Gamma_{n}^{2}\right)^{1 / 2} \text {. }
$$

It is easy to show from the basic TDHF equations that the $\Gamma_{q}, \Gamma_{A}$ are time independent after a collision and that the widths for the right- and lefthand fragments are equal.

Although formulas (6.15) - (6.21) have been derived assuming that $\rho$ arises from a single slater determinant (i.e., $\rho^{2}=\rho$ ), we assume that they are applicable in the filling approximation (Sec. VI A), when the $n_{\alpha}$ 's are noninteger. However, in the filling approximation at $t=0$, the $\Gamma_{\alpha}$ do not vanish (as they would for a pure determinant) and, moreover, are different for each ion. Indeed, from (6.18) and (6.19),

$$
\sigma_{\alpha}^{2}=\sum_{\alpha \in q} n_{\alpha}\left(1-n_{\alpha}\right),
$$

and, for the case in which we have a uniform fractional occupation $f$ of the orbitals of the last un- filled shell (as in Table II), we find that

$$
\sigma_{\alpha}^{2}=(1-f) N^{\prime},
$$

where $N^{\prime}$ is the number of nucleons in the last shell. In Table IV we list the initial widths for the static ions used in this paper.

The contribution to the width due to the filling approximation alone is an effect which persists throughout the entire TDHF calculation and we regard it as unphysical. In an attempt to roughly correct for this spurious effect, we define the quantities

$$
\bar{\Gamma}_{q}^{2}=\Gamma_{q}{ }^{2}-\left[\Gamma_{q}\left({ }^{84} \mathrm{Kr}, t=0\right)\right]^{2},
$$

and

$$
\bar{\Gamma}_{A}^{2}=\Gamma_{A}^{2}-\left[\Gamma_{A}\left({ }^{84} \mathrm{Kr}, t=0\right)\right]^{2},
$$

in which we have subtracted from the widths the contributions due to the initial ${ }^{84} \mathrm{Kr}$ ion, and the $\Gamma_{q}$ are computed for the light, $\mathrm{Kr}$-like fragment.

In the next section we will also consider the basis-dependent quantity $P_{\mathrm{Kr}}$, which is defined as the percentage of $\mathrm{Kr}$ orbitals remaining in the light fragment after the collision. ${ }^{8}$ That is, initially we have a number of orbitals which are localized in the projectile; we follow them throughout the collision, and at the end of the calculation we determine what fraction of them still remains in the scattered $\mathrm{Kr}$-like ion. This quantity is a measure of the amount of nucleon exchange between the fragments and can be calculated explicitly from the expression

$$
P_{\mathrm{Kr}}=\frac{1}{84} \sum_{\alpha}^{\prime} n_{\alpha} \omega_{\alpha \alpha},
$$

where the $\omega_{\alpha \alpha}$ are given by (6.19) and the prime on the summation indicates that only those orbitals originating in the ${ }^{84} \mathrm{Kr}$ nucleus are to be included. Of course, at $t=0$, the $\omega_{\alpha \alpha}=1$ for these orbitals, and $P_{\mathrm{Kr}}=1$.

\section{Computation of the single-particle energies}

Although not included in the results presented below, the time-dependent single-particle energies

$$
\epsilon_{\alpha}^{(n)} \equiv\left\langle\psi_{\alpha}^{(n)}\left|h^{(n)}\right| \psi_{\alpha}^{(n)}\right\rangle
$$

are quantities of interest for the incorporation of pairing ${ }^{18,62}$ and in theories attempting to extend

TABLE IV. Initial widths for ${ }^{84} \mathrm{Kr},{ }^{208} \mathrm{~Pb}$, and ${ }^{209} \mathrm{Bi}$.

\begin{tabular}{rccc}
\hline \hline Nucleus & $\Gamma_{p}$ & $\Gamma_{n}$ & $\Gamma_{A}$ \\
\hline${ }^{84} \mathrm{Kr}$ & 2.72 & 4.96 & 5.66 \\
${ }^{208} \mathrm{~Pb}$ & 5.50 & 5.99 & 8.13 \\
${ }^{209} \mathrm{Bi}$ & 5.43 & 5.99 & 8.08 \\
\hline \hline
\end{tabular}


TDHF to include two-body collisions. ${ }^{63}$ Although a straightforward evaluation of (6.26) is possible, the complexity of $h$ makes this method inefficient. Rather, since a time evolution is being performed anyway, we consider

$$
\begin{aligned}
\epsilon_{\alpha}^{(n)} \approx \epsilon_{\alpha}^{[n(1 / 2]]} & =\left\langle\psi_{\alpha}^{[n+(1 / 2)]}\left|h^{[n+(1 / 2)]}\right| \psi_{\alpha}^{[n+(1 / 2)]}\right\rangle \\
& =\left\langle\psi_{\alpha}^{[n+(1 / 2)]}\left|i \hbar \frac{\partial}{\partial t}\right| \psi_{\alpha}^{[n+(1 / 2)]}\right\rangle \\
& \approx\left\langle\frac{\psi_{\alpha}^{(n+1)}+\psi_{\alpha}^{(n)}}{2} \mid \frac{i \hbar}{\Delta t}\left(\psi_{\alpha}^{(n+1)}-\psi_{\alpha}^{(n)}\right)\right\rangle .
\end{aligned}
$$

However, since $\left\langle\psi_{\alpha}^{(n+1)} \mid \psi_{\alpha}^{(n+1)}\right\rangle=\left\langle\psi_{\alpha}^{(n)} \mid \psi_{\alpha}^{(n)}\right\rangle=1$,

$$
\epsilon_{\alpha}^{(n)} \approx-\frac{\hbar}{\Delta t} \operatorname{Im}\left\langle\psi_{\alpha}^{(n)} \mid \psi_{\alpha}^{(n+1)}\right\rangle,
$$

which is quite simple to evaluate.

\section{E. Transparency effects and mesh shifting for nearly head-on collisions}

In the results presented for very heavy-ion scattering in the next section, the Coulomb force strongly dominates the collision, with the projectile-like fragment emerging on the same side of the mesh from which the projectile originated. For the energies and angular momenta studied, we found no fusion or any indication of orbiting, although fusion has been observed for higher energies and smaller angular momenta of the ${ }^{84} \mathrm{Kr}$ $+{ }^{209} \mathrm{Bi}$ system. ${ }^{26}$ The fusion behavior of a variety of systems has been investigated (Refs. 6,7,11, $12,14,19,22,23,25,26,29,31,33,34)$.

For light-ion scattering, as the orbital angular momentum is varied at fixed energy, there are three qualitatively distinct regimes. (i) For very large $L$, there is pure Coulomb scattering, followed by a rainbow region and orbiting as $l$ decreases. (ii) There is an intermediate range of $L$ which usually fuses, although fusion disappears at sufficiently high energies. This fusion region may extend to $L=0$ or it may terminate at a nonzero $L$-value, indicating the presence of a lower angular momentum limit to fusion. ${ }^{12,23}$ (iii) If there is no fusion or if a lower angular momentum limit exists, then for the smallest $L$ values there is a region of highly inelastic scattering associated with vibrational instability. ${ }^{10}$

We will be especially concerned in this section with this last angular momentum region (iii). Early TDHF calculations were for mass-symmetric systems, ${ }^{2-6}$ so that there was no way to determine whether the incident projectile "passes through" or is "reflected" from the target. This question can be resolved by considering a massasymmetric collision. Figure 1 shows density
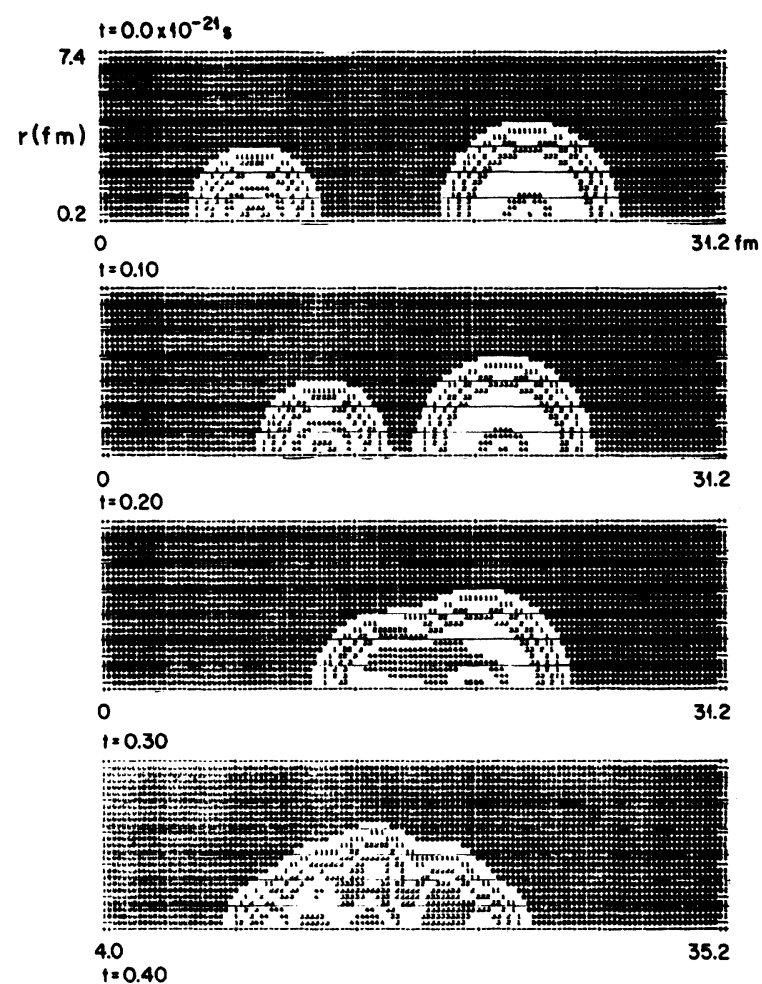

4.0
$t=0.40$

5.2

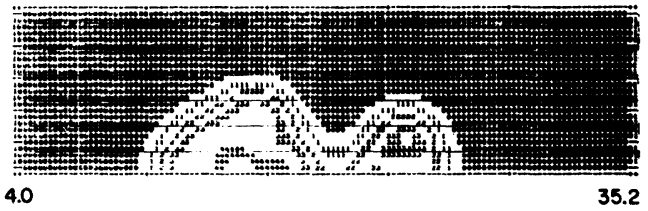

4.0

35.2

$t=0.50$

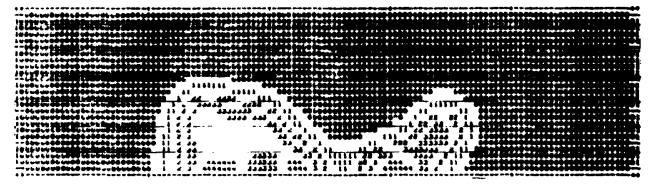

4.0

35.2

$t=0.60$

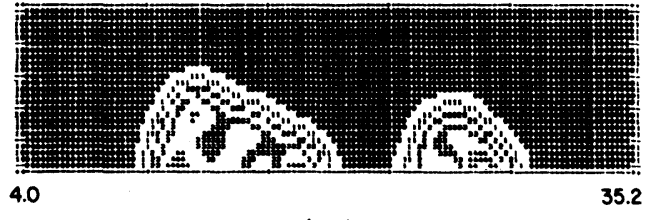

$z(f m)$

FIG. 1. Equidensity contours in the rotating frame at various times during an ${ }^{16} \mathrm{O}+{ }^{40} \mathrm{Ca}$ collision at $E_{\text {lab }}=224$ $\mathrm{MeV}$ and $l=15$. The calculation was performed using 20 mesh points in the $r$ direction, 80 mesh points in the $z$ direction, $\Delta r=\Delta z=0.40 \mathrm{fm}$, and $\Delta t=0.0025 \times 10^{-21} \mathrm{~s}$. In each case the abscissa ( $z$ axis) lies along the line joining the mass centers of the projectile and target. The axially symmetric density is plotted as a function of the cylindrical coordinates $z$ and $r$ (ordinate). The times here and in other figures are in units of $10^{-21} \mathrm{~s}$. 
contour plots for ${ }^{16} \mathrm{O}+{ }^{40} \mathrm{Ca}$ at an $L$ value in region (iii). The ${ }^{16} \mathrm{O}$ ion starts out on the left-hand side of the mesh and an ${ }^{16} \mathrm{O}$-like fragment emerges on the right, implying that the projectile passes through the target. This is in agreement with intuitive expectations based on the transparency inherent in the TDHF theory. $1,48,49,64$

The above behavior should be contrasted with that observed in the region of large $L$, where the centrifugal and Coulomb repulsion do not allow the target and projectile to strongly interpenetrate. The projectile then bounces off the target or, equivalently, the projectile fragment ends up on the same side of the mesh from which it started. This is shown in Fig. 2 for one of our ${ }^{84} \mathrm{Kr}+{ }^{209} \mathrm{Bi}$ collisions.

Figure 1 illustrates a computational difficulty which occurs for mass-asymmetric reactions in region (iii). Since the projectile-like ion passes through to the right, there may not be sufficient mesh remaining to allow the collision to be completed, and the projectile-like fragment may bump into the right-hand boundary before scission. This problem can be solved very simply as follows. After the two ions have coalesced for a reasonably long time (so that it is clear that the projectile is not going to be reflected), the mesh is uniformly shifted to the right (so the density appears to be translated to the left). In Fig. 2 such a shift of $4 \mathrm{fm}$ was performed between $t$ $=0.20 \times 10^{-21} \mathrm{~s}$ and $t=0.30 \times 10^{-21} \mathrm{~s}$, after which there is ample room for the collision to continue.

The interpretation of the final center-of-mass scattering angle $\theta_{s}$ given by (6.9) is also complicated for collisions in region (iii). Consider a head-on collision, which at $t=0$ has

$$
\theta(\text { projectile })=\pi, \quad \theta(\text { target })=0,
$$

and after the collision has

$$
\theta(\text { projectile })=0, \quad \theta(\text { target })=\pi .
$$

From Eqs. (6.2) and (6.9), $\theta_{\mathrm{in}}=\pi$ and since there is no rotation

$$
\theta_{s}=\theta_{f}=\theta_{\text {in }}=\pi \quad(L=0),
$$

which is the final angular orientation of the target. Thus, we infer that in region (iii) the center-ofmass scattering angle of the projectile-like ion is $\pm\left(\theta_{s}-\pi\right)$, where $\theta_{s}$ is calculated from Eq. (6.9). The choice of sign depends upon the actual physical situation. For relatively low-energy collisions in which the projectile is attracted as it passes through the target, there is a focusing effect, with scattering through negative angles, and the plus sign is applicable. However, for higher energy collisions there is a rapid accumulation of density as the two ions interpenetrate ${ }^{65}$ with the projectile experiencing a repulsive potential and

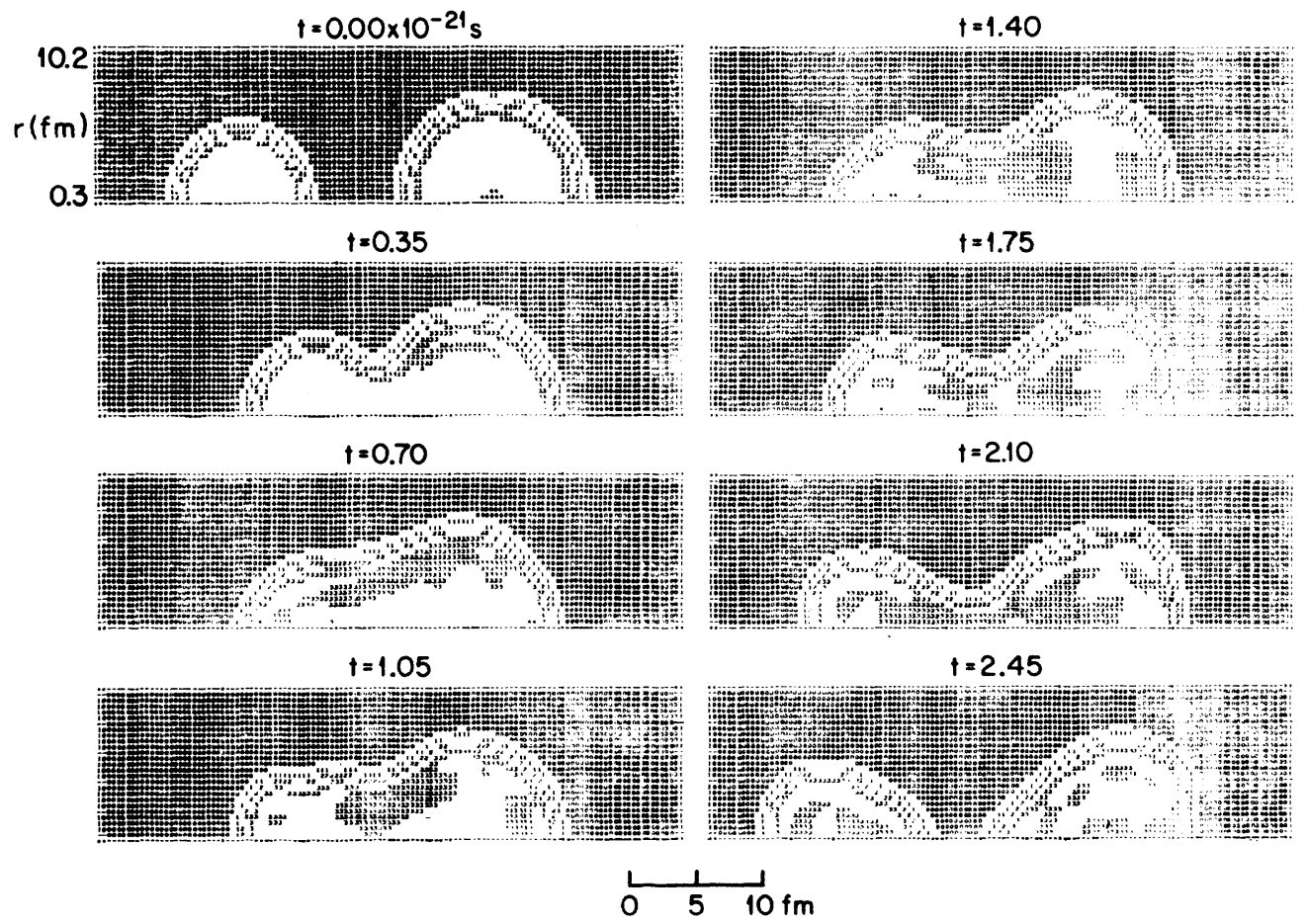

FIG. 2. Equidensity contours in the rotating frame for various times during the ${ }^{84} \mathrm{Kr}+{ }^{209} \mathrm{Bi}$ collision at $E_{\text {lab }}=714 \mathrm{MeV}$ and $l=200$. The mesh parameters are specified in Sec. VIF. 
TABLE V. Deviation of the norms from unity and variation in the total center-of-mass energy for various collisions.

\begin{tabular}{ccccc}
\hline \hline System & $\begin{array}{c}\Delta t \\
\left(10^{-21} \mathrm{~s}\right)\end{array}$ & $\begin{array}{c}\text { Typical deviation } \\
\text { of the norms } \\
\text { from unity }\end{array}$ & $\begin{array}{c}\text { Total energy } \\
(\mathrm{MeV})\end{array}$ & $\begin{array}{c}\text { Maximum } \\
\text { variation in } \\
\text { the energy }\end{array}$ \\
\hline${ }^{84} \mathrm{Kr}+{ }^{208} \mathrm{~Pb}, E_{\text {lab }}=494 \mathrm{MeV}$ & 0.0110 & $0.80 \%$ & -2020.0 & $0.60 \%$ \\
${ }^{84} \mathrm{Kr}+{ }^{209} \mathrm{Bi}, E_{\text {lab }}=600 \mathrm{MeV}$ & 0.0055 & $0.05 \%$ & -1951.0 & $0.15 \%$ \\
${ }^{84} \mathrm{Kr}+{ }^{209} \mathrm{Bi}, E_{\text {lab }}=714 \mathrm{MeV}$ & 0.0050 & $0.04 \%$ & -1868.0 & $0.10 \%$ \\
\hline \hline
\end{tabular}

scattering through positive angles, so that the negative sign is appropriate.

\section{F. Mesh parameters and numerical accuracy}

For our calculations of ${ }^{84} \mathrm{Kr}+{ }^{208} \mathrm{~Pb}$ and ${ }^{209} \mathrm{Bi}$, we used $N_{R}=20$ mesh points in the $r$ direction, and 80 mesh points in the $z$ direction $\left(N_{z}=40\right)$, with a mesh spacing $\Delta r=\Delta z=0.55 \mathrm{fm}$. These latter values were deemed adequate since detailed studies of ${ }^{40} \mathrm{Ca}+{ }^{40} \mathrm{Ca}$ indicated that increasing the mesh spacings from 0.40 to $0.55 \mathrm{fm}$ introduced errors of at most $4 \mathrm{MeV}$ in the final kinetic energy loss and $7^{\circ}$ in the scattering angle.

The stability and accuracy of calculations such as the present ones have been discussed in previous publications. $1,6,11,18,26,35$ The degree to which the single-particle norms defined by (3.3) deviate from unity during a collision is a measure of unitarity, while total energy conservation indicates the stability of the time-evolution algorithm. In Table $\mathrm{V}$ we list the time steps used in our calculations, typical percentage deviations of the norms from unity, and the maximum variations in the total center-of-mass energy during the collisions. Notice that as the velocity increases, one must be careful to decrease the time step. ${ }^{1}$ In these calculations we used 41 proton orbitals and 59 neutron orbitals. To evolve all of these 100 orbitals for one time step required approximately $17 \mathrm{~s}$ on an IBM $360 / 195$ computer. For the ${ }^{84} \mathrm{Kr}\left(E_{1 \mathrm{ab}}=600 \mathrm{MeV}\right)+{ }^{209} \mathrm{Bi}$ collision, the total number of time steps varied from about 130 for the grazing collisions to about 620 for $l=60$, the smallest $l$ value used.

\section{RESULTS}

The calculations discussed here describe the systems ${ }^{84} \mathrm{Kr}+{ }^{208} \mathrm{~Pb}$ at $E_{1 \mathrm{ab}}=494 \mathrm{MeV}$, and ${ }^{84} \mathrm{Kr}$ $+{ }^{209} \mathrm{Bi}$ at $E_{1 \mathrm{ab}}=600 \mathrm{MeV}$ and $714 \mathrm{MeV}$, for which good experimental data exist. ${ }^{45-47}$ Preliminary

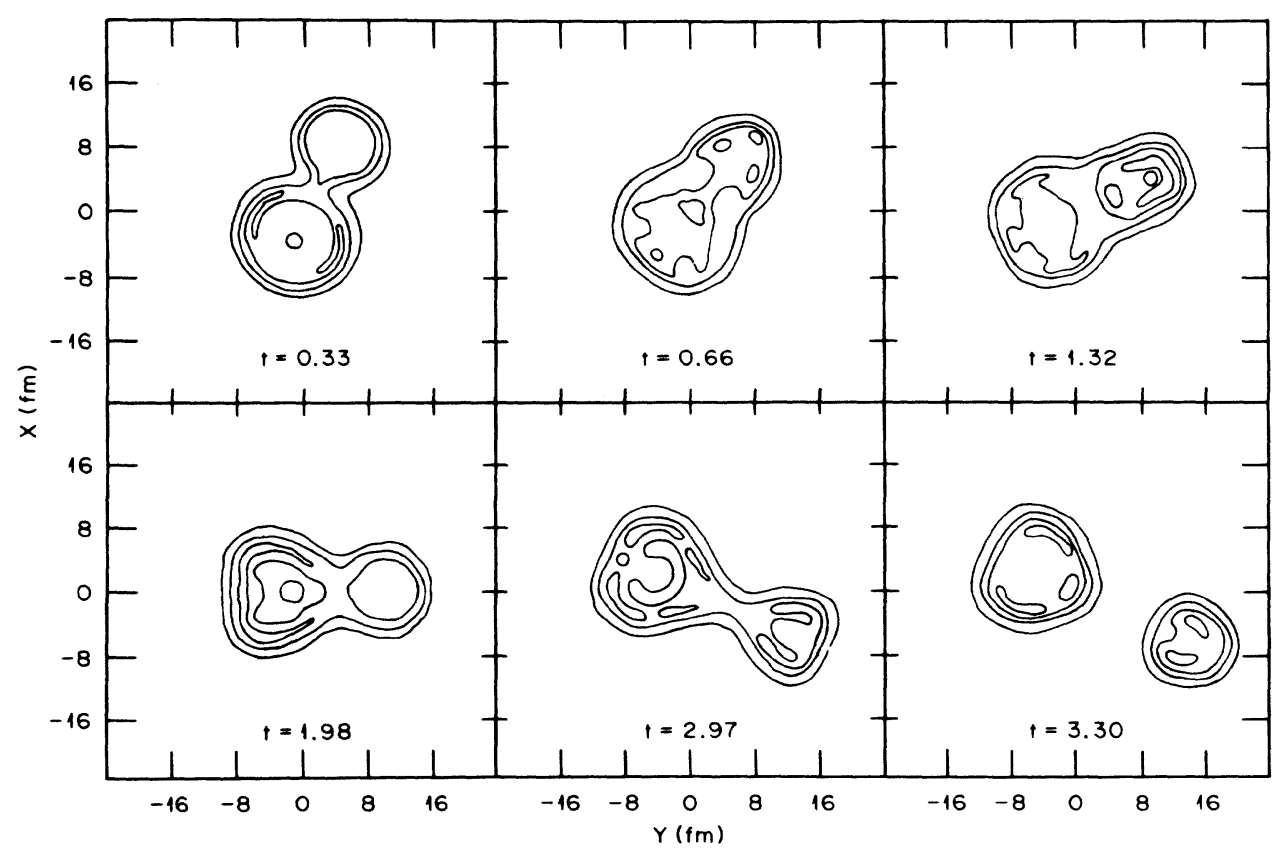

FIG. 3. Equidensity contours in the reaction plane for ${ }^{84} \mathrm{Kr}+{ }^{209} \mathrm{Bi}$ at $E_{\text {lab }}=600 \mathrm{MeV}$ and $l=140$. 


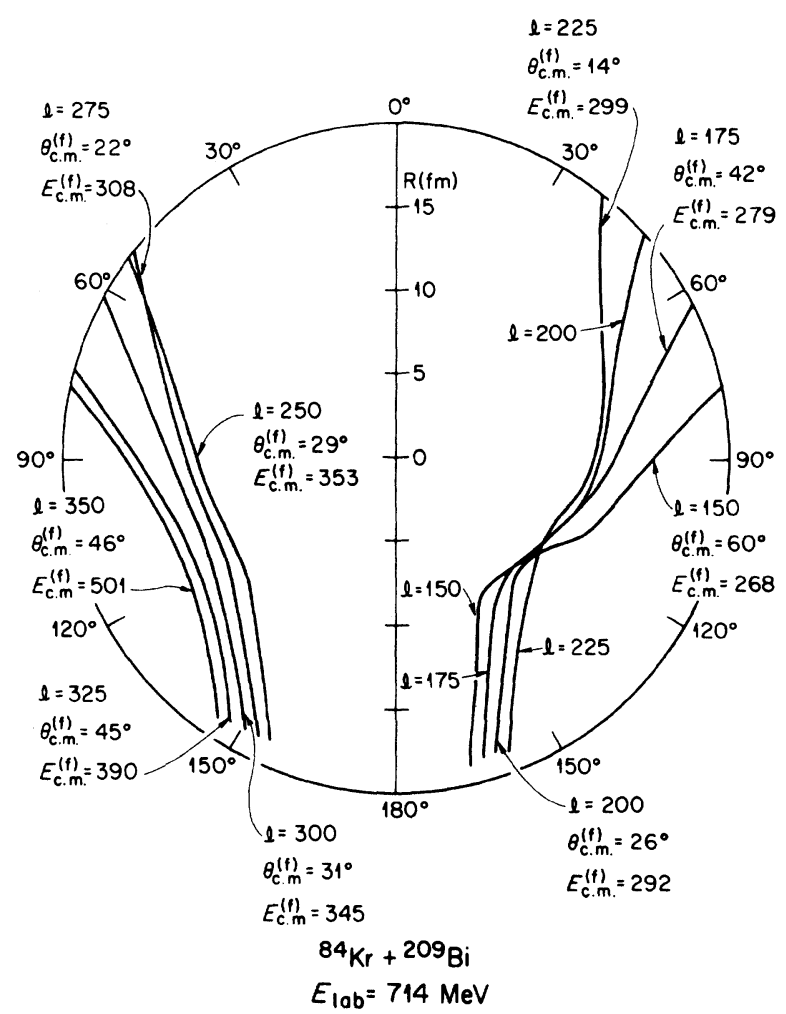

FIG. 4. $R-\theta$ trajectories for ${ }^{84} \mathrm{Kr}+{ }^{209} \mathrm{Bi}$ at $E_{\mathrm{lab}}=714$ $\mathrm{MeV}$. Note that $E_{\mathrm{c} . \mathrm{m} .}^{f}=E_{f}$ and $\theta_{\mathrm{c} . \mathrm{m} .}^{f}=\theta_{s}$ to agree with the quantities defined in Eqs. (6.10) and (6.9).

results for the 494 and $600 \mathrm{MeV}$ cases have been reported elsewhere ${ }^{8}$ and other calculations using our codes have been performed (Refs. 9, 14, 18-20, $22,23,25,26,28$ ).

The results of a typical calculation are shown in Fig. 3, which is a contour plot of the mass density in the scattering plane for the the $600 \mathrm{MeV}$ ${ }^{84} \mathrm{Kr}+{ }^{209} \mathrm{Bi}$ system at $l=140$. The grazing angular momentum for this system corresponds to

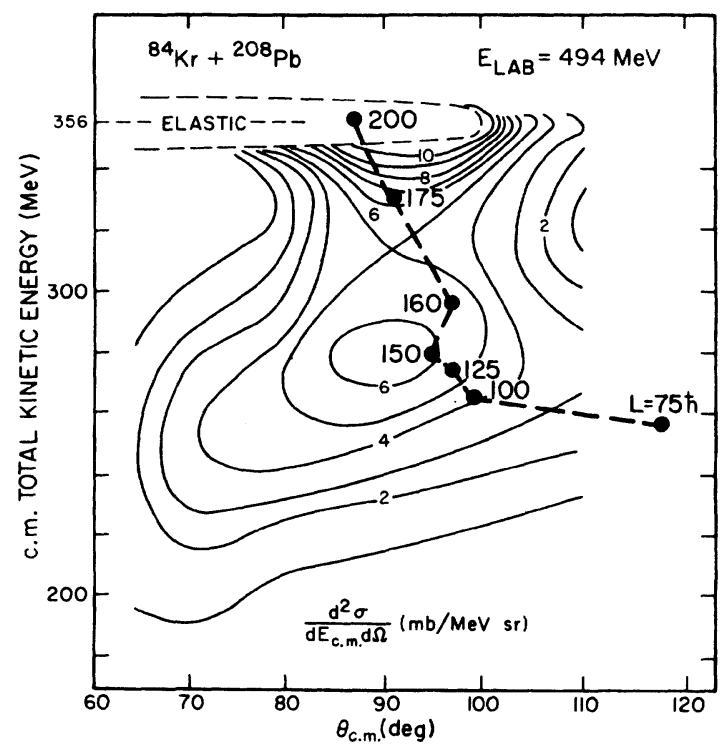

FIG. 5. Comparison of calculated points, labeled by the orbital angular momentum, with the experimental Wilczynski plot from Ref. 45 for ${ }^{84} \mathrm{Kr}+{ }^{208} \mathrm{~Pb}$ at $E_{\text {lab }}$ $=494 \mathrm{MeV}$. In this and the next two figures, the ordinate is $E_{f}$ and the abscissa is $\theta_{\text {c.m. }}=\theta_{s}$.

$l \sim 300$, so that this is a moderately central collision. Of particular interest is the fact that the ions distinctly retain their identity throughout the collision, and that the interior density is largely constant, with some fluctuations due to the motion of particular single-particle orbitals. As a result, the dinuclear system strongly resembles the picture invoked in the more phenomenological models of such reactions, i.e., independent nucleons moving between two roughly flat potential wells connected by a neck. ${ }^{66}$ Other features of interest are the near constancy of the surface diffuseness throughout the collision and the compact shape of the system at scission, the latter being

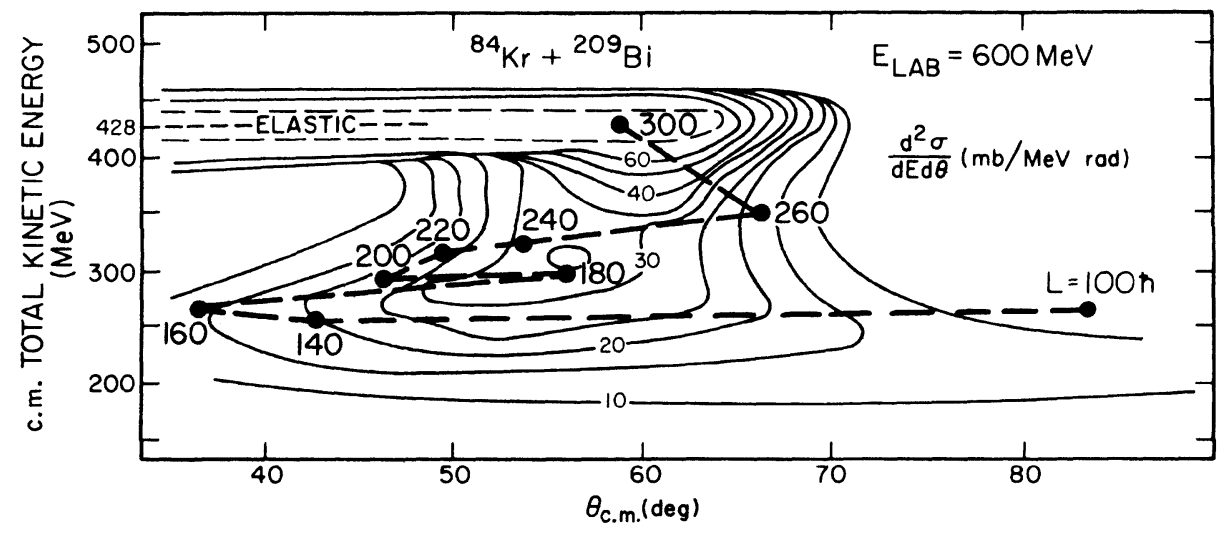

FIG. 6. Similar to Fig. 5 for ${ }^{84} \mathrm{Kr}+{ }^{209} \mathrm{Bi}$ at $E_{\mathrm{lab}}=600 \mathrm{MeV}$, with data from Ref. 46. We take the opportunity here to correct the misplotting of the $l=100$ point in our previously published Fig. 2 of Ref. 8 . 


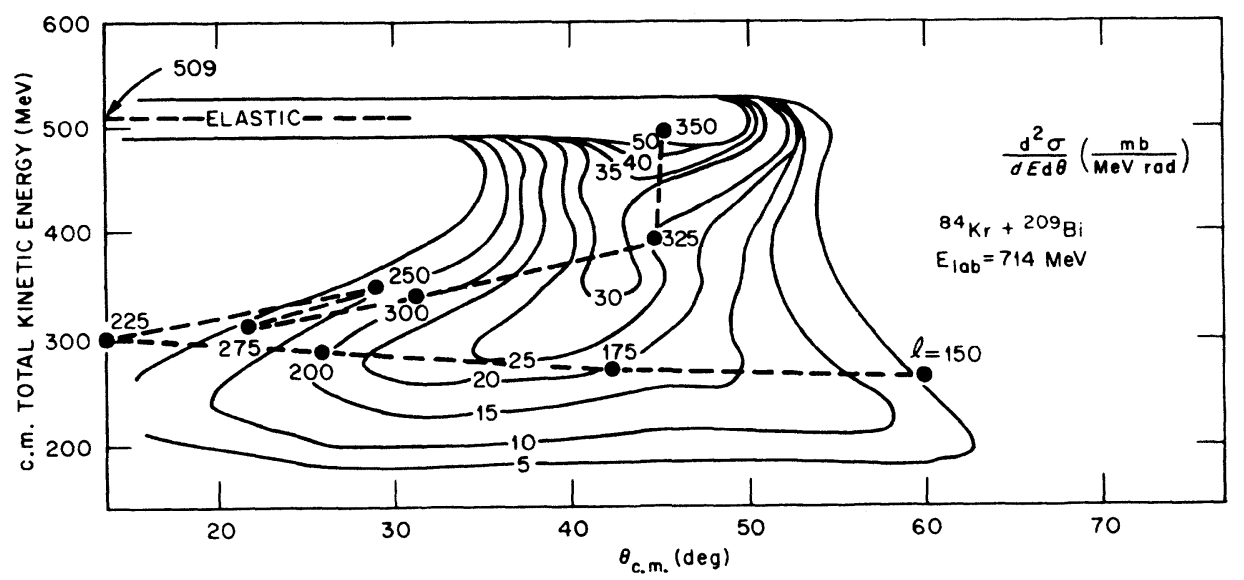

FIG. 7. Similar to Fig. 5 for ${ }^{84} \mathrm{Kr}+{ }^{209} \mathrm{Bi}$ at $E_{\text {lab }}=714 \mathrm{MeV}$, with data from Ref. 46.

in accord more with the predictions of one-body dissipation than with two-body dissipation, ${ }^{18,67}$ as expected from a TDHF calculation. These aspects are also evident for $E_{1 \mathrm{ab}}=714 \mathrm{MeV}$ at $l=200$ in the more detailed Fig. 2. It should also be remarked that at the moment of scission the radial kinetic energy $\left(\frac{1}{2} \mu_{f} \dot{R}_{f}{ }^{2}\right)$ of the system in Fig. 3 is small ( $15 \mathrm{MeV}^{2}$, to be compared with $\sim 90 \mathrm{MeV}$ at the time of initial contact), indicating strong damping during the collision.

In Fig. 4 we summarize the global behavior of the $714 \mathrm{MeV}$ system by showing trajectories in the $R-\theta$ plane. The behavior of the other systems is similar. Particularly evident are the Coulomb dominated peripheral region and the rather sharply defined minimum radius for the more central collisions, although this latter may be a result of the inadequacy of our separation coordinate $R$ for more compact shapes. It is also clear that the more central collisions can be described as a twostage process: a rapid initial approach phase with substantial ion-ion interaction and strong damping at the minimum value of $R$, followed by very slow elongation and rotation of the system.

Figures 5-7 compare our calculated final fragment kinetic energies and scattering angles with experimental Wilczyński plots. ${ }^{45-47}$ Although it is not possible to compute double differential cross sections with the present TDHF method, it is clear that the calculations qualitatively reproduce the overall behavior of the experimental data. In particular, at the two lower energies, there is the proper amount of damping in the correct angular region, with many partial waves concentrating to give a strong focusing in angle and energy near the experimental peak. For angular momenta just below grazing, the scattering angle is more forward than Rutherford and then increases to $180^{\circ}$ for small $l$. In the $600-\mathrm{MeV}$ system, there is also an inner rainbow and a pronounced fluctuation near $\theta_{\text {c. } m .}=\theta_{s}=50^{\circ}$ arising from single-particle effects (see below). The behavior of the $714-\mathrm{MeV}$ sys tem is similar to that at $600 \mathrm{MeV}$, and the much broader angular structure is reproduced, although the calculated trajectories are a bit too far forward relative to the data.

Somewhat disturbing at the small impact parameters for $600 \mathrm{MeV}$ and over a broader range of $l$ values at $714 \mathrm{MeV}$ is the lack of sufficient energy damping. This feature, although not present for heavier systems, ${ }^{20,28}$ is also evident in calculations ${ }^{19}$ performed for ${ }^{86} \mathrm{Kr}+{ }^{139} \mathrm{La}$ and might be associated with a too-compact scission shape in the TDHF calculations. However, the situation is complicated by ambiguities in the experimental data. In the $600 \mathrm{MeV} \mathrm{Kr}+\mathrm{Bi}$ system, the assumption that the average light fragment mass is 84 at all angles ignores the drift toward mass symmetry at back angles and hence results in a spurious decline of the back-angle ridge, which is known from coincidence measurements to be at a nearly constant kinetic energy. ${ }^{46}$ In the $\mathrm{Kr}$ + La systems, the mass distribution for the most strongly damped events shows two components, one centered around the projectile mass and one around symmetry. ${ }^{68}$ This indicates the presence of a fusion-fission component, not included in TDHF, which would tend to shift the experimental mass-integrated kinetic energy to lower values. Unambiguous data are probably needed before this point can be decisively settled.

In Fig. 8, we show various final-state quantities as functions of impact parameter for the three systems we have studied. The deflection functions are in qualitative agreement with elementary expectations and more detailed phenomenology. ${ }^{47}$

The final center-of-mass fragment total kinetic energy $E_{f}=T_{\text {c.m. }}$ decreases as $l$ decreases, with nonperipheral collisions resulting in damping down to or below the experimental Coulomb bar- 


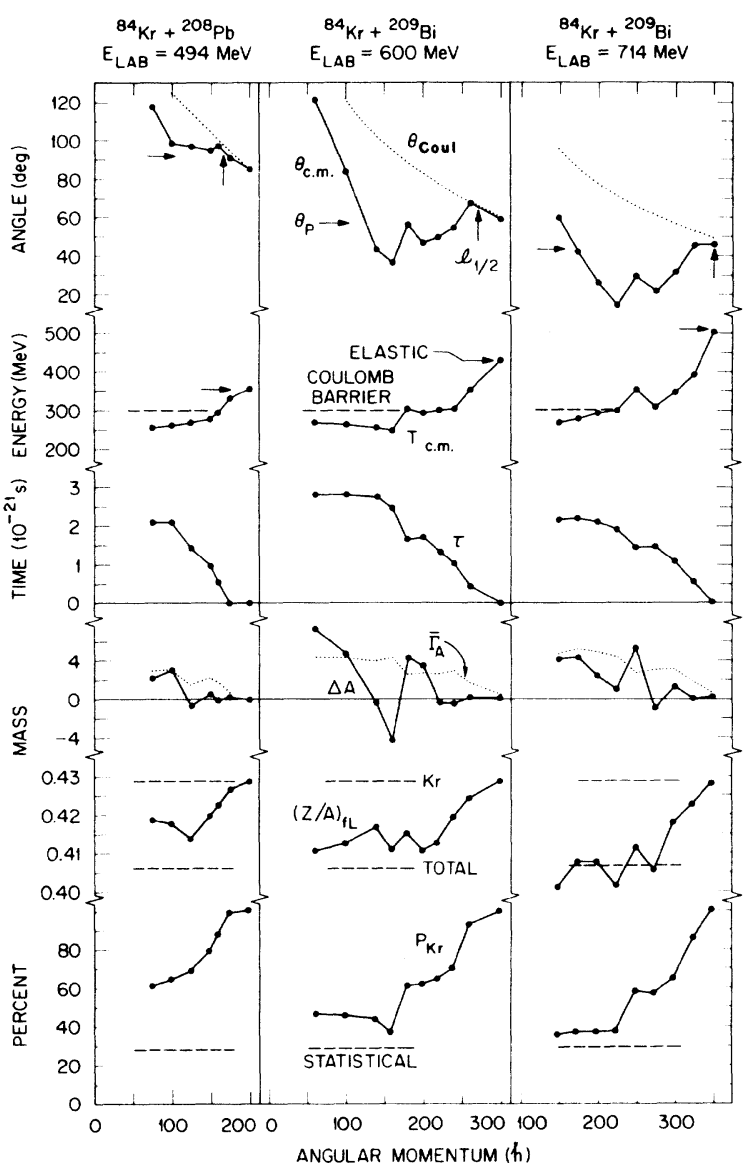

FIG. 8. Quantitative summary of TDHF results for $\mathrm{Kr}$-induced collisions. Plotted as functions of the initial orbital angular momentum are $\theta_{\text {c.m. }}=\theta_{s}$, the final c.m. scattering angle; $T_{\text {c.m. }}=E_{f}$, the final c.m. total kinetic energy of the fragments; $\tau$, the contact time; $\Delta A$, the net mass change of the light fragment; $\overline{\boldsymbol{\Gamma}}_{A}$, the corrected full width at half maximum of the mass distribution; $(Z / A)_{f L}$, the final charge-to-mass ratio of the light fragment, and $P_{\mathrm{Kr}}$, the percentage of $\mathrm{Kr}$ orbitals remaining in the light fragment after the collision. Also shown on the $\theta_{\text {c.m. }}$ graph are $\theta_{\text {Coul }}$, the Rutherford deflection function; $l_{1 / 2}$, the angular momentum where the optical-model transmission coefficients fall to $\frac{1}{2}$ (Refs. 45 and 47 ); and $\theta_{p}$, the peak angle in the experimental strongly damped angular distribution (Ref. 47). The Coulomb barriers indicated on the $\boldsymbol{T}_{\text {c.m. }}$ graphs correspond to point charges separated by $14.28 \mathrm{fm}$, the experimental (Ref. 47) strong-absorption radius for ${ }^{84} \mathrm{Kr}$ $+{ }^{209} \mathrm{Bi}$ at $E_{\mathrm{lab}}=600 \mathrm{MeV}$. Also shown are the values of $(Z / A)$ for the $\mathrm{Kr}$ ion and the composite system, and the statistical value of $P_{\mathrm{Kr}}$.

rier, independent of bombarding energy. This is, of course, a consequence of fragment deformation in the scission configuration. ${ }^{26}$

The contact time $\tau$ is defined as the interval during which the minimum density along the symmetry axis between the fragments, $\rho_{\min }$, exceeds

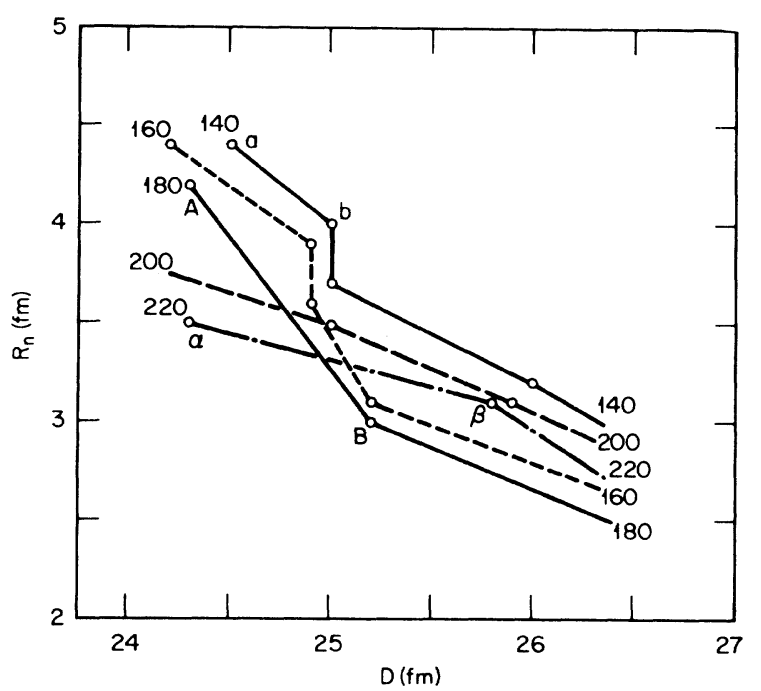

FIG. 9. Trajectories in the $R_{n}-D$ plane for ${ }^{84} \mathrm{Kr}+{ }^{209} \mathrm{Bi}$ at $E_{\text {lab }}=600 \mathrm{MeV}$ and for various $l$ values. The open circles indicate values of $R_{n}$ and $D$ at intervals of 3.3 $\times 10^{-22} \mathrm{~s}$.

$\frac{1}{2}$ of nuclear matter density. The contact time increases strongly with decreasing $l$. Thus, although smaller angular velocities are associated with the lower angular momenta, longer contact times cause the combined system to rotate through a larger angle. The fluctuations in the $\mathrm{Kr}+\mathrm{Bi}$ deflection functions are then naturally correlated with the unusually short contact times of these collisions.

The deviation of the mean mass number of the Kr-like fragment from 84, $\Delta A=A_{f L}-84$, and the full width at half maximum of the fragment mass distribution, $\bar{\Gamma}_{A}$, are displayed in Fig. 8 . In the strongly damped region at $600 \mathrm{MeV}$ centered around a scattering angle of $50^{\circ}, A_{f L}$ fluctuates around the observed value ${ }^{46}$ of 84 . At very low impact parameters, $A_{f L}$ increases significantly, although the calculated value of roughly 90 at $\theta_{c_{0} m .}=85^{\circ}$ is of insufficient magnitude to agree with the experimental value of 110 . The width $\bar{\Gamma}_{A}$ is always an order of magnitude smaller than the observed value of roughly 30 , and is smaller than the limit imposed by the use of a determinantal wave function. ${ }^{69}$

The final charge-to-mass ratio of the light fragment, $(Z / A)_{f L}$, is also shown in Fig. 8. For peripheral collisions, it is nearly equal to that of the original $\mathrm{Kr}$ ion, but it decreases toward a value characteristic of the composite system with decreasing $l$.

The quantity $P_{\mathrm{Kr}}$ (which measures the amount of single-particle interchange between the fragments) is unity for peripheral collisions, but decreases dramatically for the smaller impact parameters, 


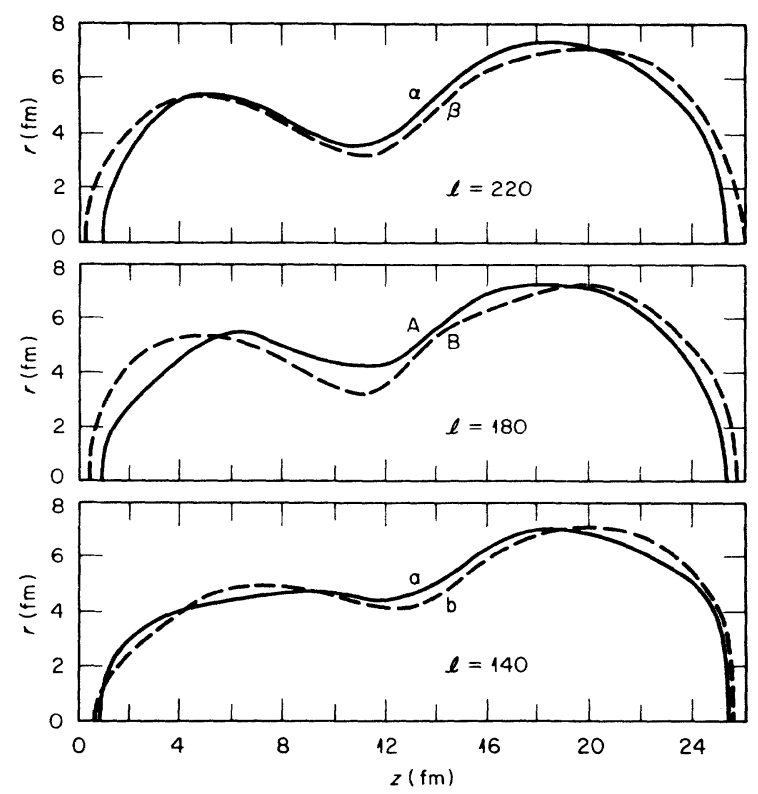

FIG. 10. Half-density contours in the rotating frame for ${ }^{84} \mathrm{Kr}+{ }^{209} \mathrm{Bi}$ at $E_{\text {lab }}=600 \mathrm{MeV}$ and $l=140,180$, and 220. The solid contour denotes the shape corresponding to $D \approx 24.5 \mathrm{fm}$ and the dashed curve represents the shape $3.3 \times 10^{-22} \mathrm{~s}$ later. The curves $\alpha, \beta, A, B, a$, and $b$ correspond to the points labeled in Fig. 9 .

indicating substantial mixing of the orbitals in these collisions. For the most central collisions, $P_{\mathrm{Kr}}$ is nearly equal to the value expected if nucleons were distributed statistically between the two nuclei. ${ }^{70}$

In view of this underlying single-particle mechanism, it is not surprising that fluctuations will arise in $\tau$, and hence in $\theta_{s}$, due to the vagaries of the orbitals in the neck region. To see this, consider, for example, the detailed evolution of the shape of the system. Let $D$ be the distance along the symmetry axis between the half density points of the outer fragment surfaces and $R_{n}$ be the half-density radius at the location of the neck, $z=z_{\mathrm{min}}$. The trajectories in the $R_{n}-D$ plane shown in Fig. 9 imply that the stretching and scission motion of the system has a strong $l$ dependence. The trajectories plotted correspond only to the elongation from the most compact shape, so that $D$ is a monotonically increasing function of time. For the most compact shapes, $R_{n}$ systematically increases with decreasing $l$, as is expected from macroscopic considerations. However, at $D=25$ fm, an abrupt crossover occurs, with the neck radius decreasing quite suddenly for $l=180$ and also less rapidly for 160 . These trends are also evident in Fig. 10, which shows half-density contours at time intervals of $3.3 \times 10^{-22} \mathrm{~s}$ for the times $A, B, a, b, \alpha$, and $\beta$ indicated on Fig. 9.
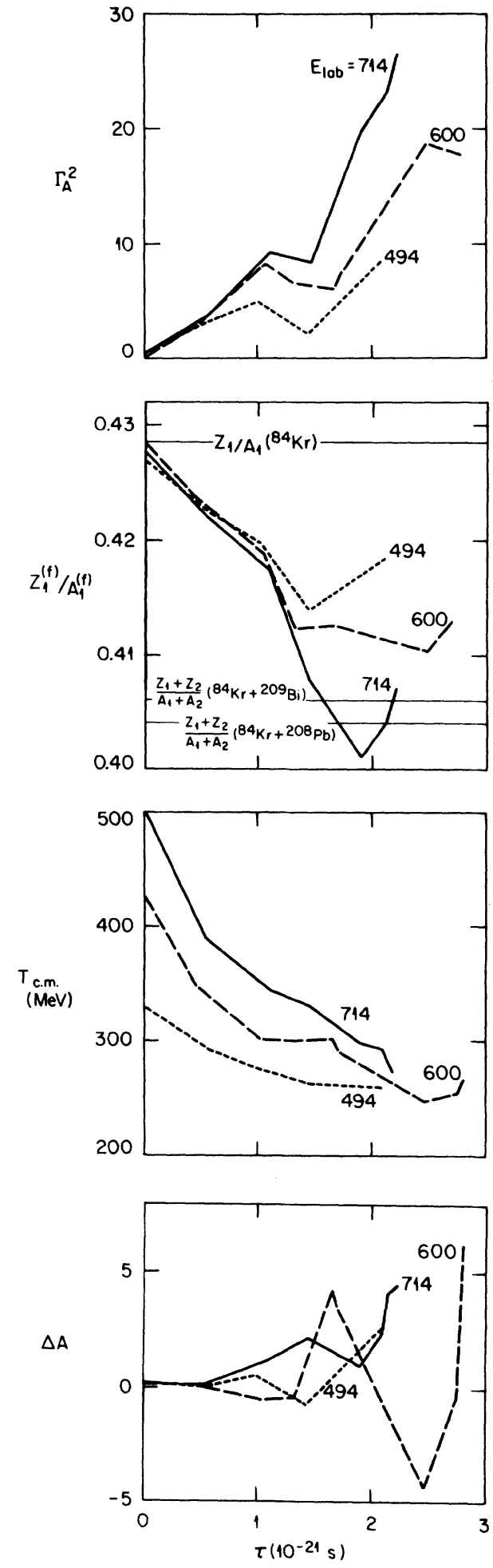

FIG. 11. Summary of various final-state quantities as functions of the contact time $\tau$. To agree with the definitions in Sec. VI, we note that $\Gamma_{A}=\bar{\Gamma}_{A}, Z_{\mathbf{i}}^{(f)}=Z_{f L}$, $A_{1}^{(f)}=A_{f L}, Z_{1}=Z_{L}, Z_{2}=Z_{R}, A_{1}=A_{L}, A_{2}=A_{R}$, and $\mathrm{T}_{\text {c.m. }}$. $=E_{f}$. 
These contours also display the trends in the average macroscopic shape of the system at different angular momenta. In summary, it is not unlikely that the fluctuations in $\tau$ and $\theta_{s}$ are due to a premature scission induced by a "snapping" of the neck.

Various phenomenological treatments make predictions about how certain final-state quantities depend upon the contact time. ${ }^{47}$ It is therefore of interest to consider such correlations which may exist in our TDHF calculations. To this end, Fig. 11 displays $\bar{\Gamma}_{A}^{2}, Z_{f L} / A_{f L}$, the total fragment kinetic energy, and $\Delta A$ as functions of $\tau$ for our three systems. The energy loss increases monotonically with $\tau$, rapidly at first and then at a slower rate, consistent with the behavior inferred from the experimental correlation between $T_{\text {c.m. }}$. and the width of the fragment charge distribution. The rate of charge-to-mass equilibration seems to be independent of energy for $\tau \lesssim 1.5 \times 10^{-21} \mathrm{~s}$ but fully equilibrates to the value for the combined system only at $714 \mathrm{MeV}$, probably due to the greater interpenetration at higher energies. There is also some indication of an overshoot at this last energy for $\tau \sim 2 \times 10^{-21} \mathrm{~s}$. The roughly linear dependence of $\bar{\Gamma}_{A}^{2}$ on $\tau$ is consistent with diffusion models, although the diffusion coefficients $\bar{\Gamma}_{A}{ }^{2} / 16 \tau \ln 2=0.4,0.6$, and $0.9(\mathrm{amu})^{2} /$ $10^{-21} \mathrm{~s}$, in the order of increasing energy, are significantly small. ${ }^{47}$

\section{ACKNOWLEDGMENTS}

We wish to acknowledge helpful discussions with V. Maruhn-Rezwani, J. W. Negele, and R. Vandenbosch. We thank J. Maruhn for providing a fast input/output code for the IBM-360 system. This research was supported in part by the Division of Basic Energy Sciences, U.S. Department of Energy, under Contract No. W-7405-eng-26 with the Union Carbide Corporation, and by the National Science Foundation under Contracts No. PHY77-21602 and PHY79-23638. S. E. Koonin is an Alfred P. Sloan Foundation Fellow.
${ }^{1}$ P. Bonche, S. E. Koonin, and J. W. Negele, Phys. Rev. C 13, 1226 (1976).

${ }^{2}$ S. E. Koonin, Phys. Lett. B61, 227 (1976).

${ }^{3}$ R. Y. Cusson and J. A. Maruhn, Phys. Lett. B62, 134 (1976).

${ }^{4} \mathrm{~J}$. A. Maruhn and R. Y. Cusson, Nucl. Phys. A270, 471 (1976).

${ }^{5}$ R. Y. Cusson, R. K. Smith, and J. A. Maruhn, Phys. Rev. Lett. 36, 1166 (1976).

${ }^{6}$ S. E. Koonin, K. T. R. Davies, V. Maruhn-Rezwani, H. Feldmeier, S. J. Krieger, and J. W. Negele, Phys. Rev. C 15, 1359 (1977).

${ }^{7}$ V. Maruhn-Rezwani, K. T. R. Davies, and S. E. Koonin, Phys. Lett. B67, 134 (1977).

${ }^{8}$ K. T. R. Davies, V. Maruhn-Rezwani, S. E. Koonin, and J. W. Negele, Phys. Rev. Lett. 41, 632 (1978).

${ }^{9}$ A. K. Dhar and B. S. Nilsson, Phys. Lett. B77, 50 (1978).

${ }^{10}$ R. Y. Cusson, J. A. Maruhn, and H. W. Meldner, Phys. Rev. C 18, 2589 (1978).

${ }^{11}$ H. Flocard, S. E. Koonin, and M. S. Weiss, Phys. Rev. C 17,1682 (1978).

${ }^{12} \mathrm{P}$. Bonche, B. Grammaticos, and S. E. Koonin, Phys Rev. C 17, 1700 (1978).

${ }^{13}$ K. T. R. Davies, H. T. Feldmeier, H. Flocard, and M. S. Weiss, Phys. Rev. C 18, 2631 (1978)

${ }^{14}$ S. J. Krieger and K. T. R. Davies, Phys. Rev. C 18 , 2567 (1978).

${ }^{15}$ S. E. Koonin, B. Flanders, H. Flocard, and M. S. Weiss, Phys. Lett. B77, 13 (1978).

${ }^{16}$ K. R. Sandhya Devi and M. R. Strayer, J. Phys. G $\underline{4}$, L97 (1978).

${ }^{17}$ K. R. Sandhya Devi and M. R. Strayer, Phys. Lett. B77, 135 (1978).

${ }^{18} \mathrm{~J}$. W. Negele, S. E. Koonin, P. Möller, J. R. Nix, and
A. J. Sierk, Phys. Rev. C 17, 1098 (1978).

${ }^{19}$ K. T. R. Davies, K. R. Sandhya Devi, and M. R. Strayer, Phys. Rev. C 20, 1372 (1979).

${ }^{20}$ A. K. Dhar, Phys. Rev. Lett. $\underline{42}$, 1124 (1979).

${ }^{21}$ R. Y. Cusson and H. W. Meldner, Phys. Rev. Lett. $\underline{42}$, 694 (1979).

${ }^{22}$ S. J. Krieger and K. T. R. Davies, Phys. Rev. C 20, 167 (1979).

${ }^{23}$ P. Bonche, K. T. R. Davies, B. Flanders, H. Flocard, B. Grammaticos, S. E. Koonin, S. J. Krieger, and M. S. Weiss, Phys. Rev. C 20, 641 (1979).

${ }^{24}$ K. R. Sandhya Devi, M. R. Strayer, and J. M. Irvine, J. Phys. G $\underline{5}, 281$ (1979).

${ }^{25}$ A. K. Dhar and B. S. Nilsson, Nucl. Phys. A315, 445 (1979).

${ }^{26}$ K. T. R. Davies, K. R. Sandhya Devi, and M. R. Strayer, Phys. Rev. Lett. 44, 23 (1980) and unpublished.

${ }^{27}$ R. Y. Cusson, J. A. Maruhn, and H. Stöcker, Z. Phys. A 294,257 (1980).

${ }^{28}$ A. K. Dhar, B. S. Nilsson, K. T. R. Davies, and S. E. Koonin, Nucl. Phys. (to be published).

${ }^{29}$ B. Flanders, P. Bonche, S. E. Koonin, and M. S. Weiss (unpublished).

${ }^{30}$ R. Y. Cusson, J. A. Maruhn, W. Greiner, and H. Stöcker (unpublished).

${ }^{31}$ J. Hahn, R. Y. Cusson, H. Stöcker, H. J. Lustig, J. A. Maruhn, and W. Greiner (unpublished).

${ }^{32}$ H. Stöcker, R. Y. Cusson, J. A. Maruhn, and W. Greiner (unpublished).

${ }^{33}$ K. R. Sandhya Devi, M. R. Strayer, J. M. Irvine, and K. T. R. Davies, Phys. Rev. C 23, 1064 (1981).

${ }^{34}$ K. R. Sandhya Devi, A. K. Dhar, and M. R. Strayer (unpublished).

${ }^{35}$ P. Hoodbhoy and J. W. Negele, Nucl. Phys. A288, 23 (1977). 
${ }^{36}$ P. Quentin and H. Flocard, Annu. Rev. Nucl. Sci. $\underline{28}$, $523(1978)$ and references cited therein.

${ }^{37}$ S. Levit, Phys. Rev. C 21, 1594 (1980).

${ }^{38} \mathrm{~S}$. Levit, J. W. Negele, and Z. Paltiel, Phys. Rev. C 21, 1603 (1980).

${ }^{39}$ S. Levit, J. W. Negele, and Z. Paltiel, MIT Report No. CTP 836 (unpublished).

${ }^{40}$ Y. Alhassid and S. E. Koonin (unpublished).

${ }^{41}$ Y. Alhassid, B. Müller, and S. E. Koonin, Phys. Rev. C 23, 487 (1981).

${ }^{42} \mathrm{~J} . \overline{\mathrm{W}}$. Negele, private communication.

${ }^{43} \mathrm{~V}$. Maruhn-Rezwani, N. Grün, and W. Scheid, Phys. Rev. Lett. 43, 512 (1979).

${ }^{44}$ K. R. Sandhya Devi, S. E. Koonin, and Y. Alhassid (unpublished).

${ }^{45}$ R. V andenbosch, M. P. Webb, and T. D. Thomas, Phys. Rev. Lett. 36, 459 (1976); Phys. Rev. C 14, 143 (1976).

${ }^{46}$ K. L. Wolf, J. P. Unik, J. R. Huizenga, J. Birkelund, H. Freiesleben, and V. E. Viola, Phys. Rev. Lett. 33, 1105 (1974); K. L. Wolf and C. T. Roche, in Proceedings of the Symposium on Macroscopic Features of Heavy-Ion Collisions, Argonne, Mlinois, 1976, edited by D. G. Kovar, ANL Report No. ANL/PHY-76-2 (unpublished), Vol. I, p. 295.

${ }^{47}$ W. U. Schröder and J. R. Huizenga, Annu. Rev. Nucl. Sci. 27, 465 (1977).

${ }^{48} \mathrm{~J}$. W. Negele, in Theoretical Methods in Medium-Energy and Heavy Ion Physics, edited by K. W. McVoy and W. A. Friedman (Plenum, New York, 1978), p. 235.

${ }^{49}$ S. E. Koonin, in Progress in Particle and Nuclear Physics, edited by D. Wilkinson (Pergamon, Oxford, 1980), Vol. 4, pp. 283-321.

${ }^{50}$ A. K. Kerman and S. E. Koonin, Ann. Phys. (N.Y.) 100 , 332 (1976).

${ }^{51}$ Y. M. Engel, D. M. Brink, K. Goeke, S. J. Krieger, and D. Vautherin, Nucl. Phys. A249, 215 (1975).

${ }^{52} \mathrm{~J}$. W. Negele and D. Vautherin, Phys. Rev. C $\underline{5}, 1472$ (1972).

${ }^{53}$ Y. Boneh and Z. Fraenkel, Phys. Rev. C 10, 893 (1974); Y. Boneh, Lawrence Berkeley Laboratory Report, 1975 (unpublished).
${ }^{54}$ R. Varga, Matrix Iterative Analysis (Prentice-Hall, Englewood Cliffs, 1962), p. 195.

${ }^{55} \mathrm{R}$. V arga, Matrix Iterative Analysis (Prentice-Hall, Englewood Cliffs, 1962), p. 273.

${ }^{56} \mathrm{E}$. L. Wachspress, Iterative Solution of Elliptic Systems and Applications to the Neutron Diffusion Equations of Reactor Physics (Prentice-Hall, Englewood Cliffs, 1966) p. 226.

${ }^{57}$ A. R. Mitchell, Computational Methods in Partial Differential Equations (Wiley, New York, 1969), p. 61.

${ }^{58}$ K. T. R. Davies, H. Flocard, S. J. Krieger, and M. S. Weiss, Nucl. Phys. A342, 111 (1980).

${ }^{59} \mathrm{C}$. W. De Jager, H. DeVries, and C. DeVries, At. Data Nucl. Data Tables 14, 479 (1974).

${ }^{60}$ A. H. Wapstra and K. Bos, At. Data Nucl. Data Tables 19,177 (1977).

${ }^{61} \mathrm{H}$. Goldstein, Classical Mechanics (Addison-Wesley, Cambridge, 1953), p. 76.

${ }^{62} \mathrm{~J}$. Blocki and H. Flocard, Nucl. Phys. A273, 45 (1976).

${ }^{63}$ C. Y. Wong and H. H. K. Tang, Phys. Rev. Lett. $\underline{40}$, 1070 (1978); C. Y. Wong and H. H. K. Tang, Phys. Rev. C 20, 1419 (1979); C. Y. Wong and K. T. R. Davies, Phys. Lett. B96, 258 (1980); C. M. Shakin and M. S. Weiss, UCRL Report No. 8500 (unpublished); H. Orland and R. Schaeffer, Z. Phys. A 290, 191 (1979).

${ }^{64} \mathrm{H}$. S. Köhler and H. Flocard, Nucl. Phys. A323, 189 (1979).

${ }^{65} \mathrm{C}$. Y. Wong and K. T. R. Davies (unpublished).

${ }^{66} \mathrm{~J}$. Randrup, Nucl. Phys. A307, 319 (1978); $\underline{\text { A327 }}, 490$ (1979).

${ }^{67}$ J. Błocki, Y. Boneh, J. R. Nix, J. Randrup, M. Robel, A. J. Sierk, and W. J. Swiatecki, Ann. Phys. (N.Y.) 113,330 (1978).

${ }^{68}$ P. Dyer, M. P. Webb, R. J. Puigh, R. Vandenbosch, T. D. Thomas, and M. S. Zisman, Phys. Rev. C $\underline{22}$, 1509 (1980).

${ }^{69}$ C. H. Dasso, T. Døssing, and H. C. Pauli, Z. Phys. A 289, 395 (1979).

${ }^{70} \mathrm{H}$. Feldmeier, invited lecture presented at the Twelfth Summer School on Nuclear Physics: Nuclear Structure Study by Means of Nuclear Reactions, 1979, Mikołajki, Poland, to be published in Nukleonika. 


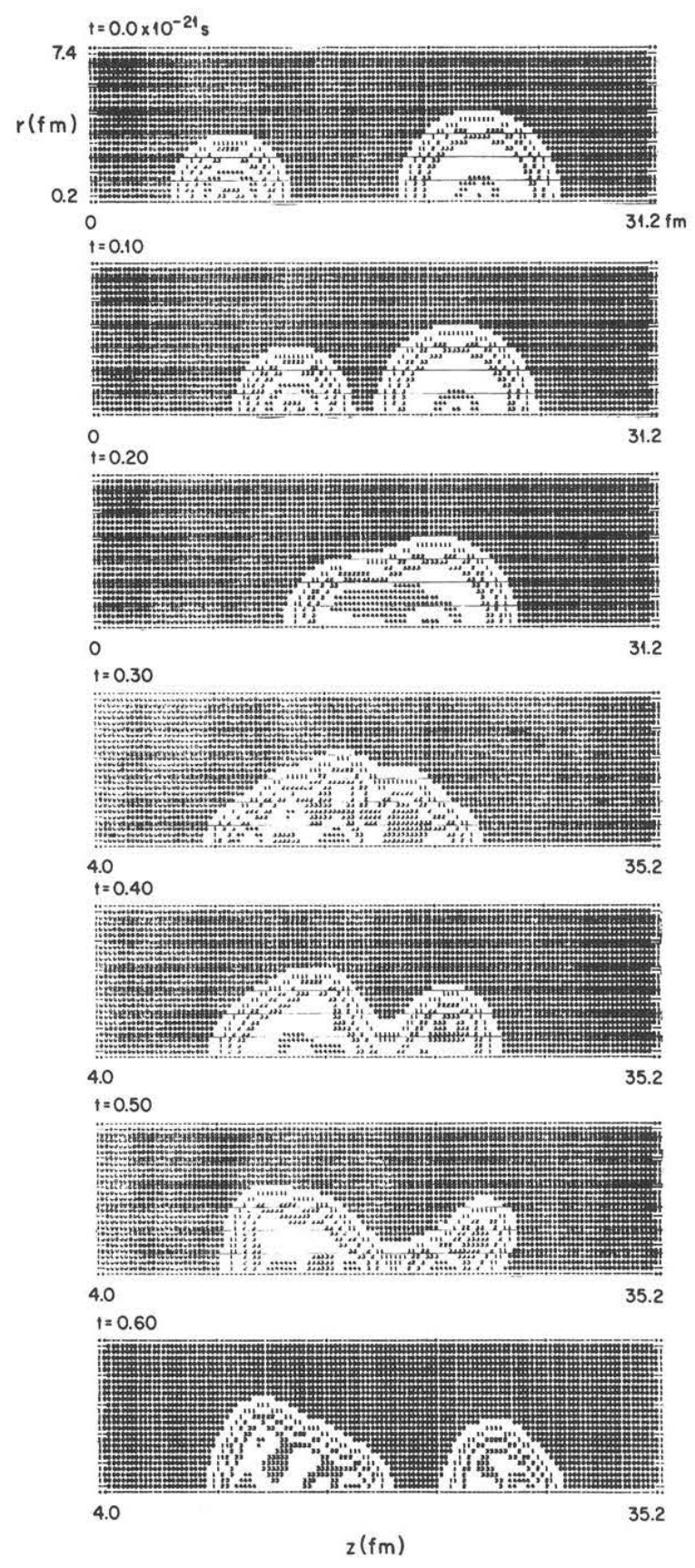

FIG. 1. Equidensity contours in the rotating frame at various times during an ${ }^{16} \mathrm{O}+{ }^{40} \mathrm{Ca}$ collision at $E_{\text {lab }}=224$ $\mathrm{MeV}$ and $l=15$. The calculation was performed using 20 mesh points in the $r$ direction, 80 mesh points in the $z$ direction, $\Delta r=\Delta z=0.40 \mathrm{fm}$, and $\Delta t=0.0025 \times 10^{-21} \mathrm{~s}$. In each case the abscissa ( $z$ axis) lies along the line joining the mass centers of the projectile and target. The axially symmetric density is plotted as a function of the cylindrical coordinates $z$ and $r$ (ordinate). The times here and in other figures are in units of $10^{-21} \mathrm{~s}$. 

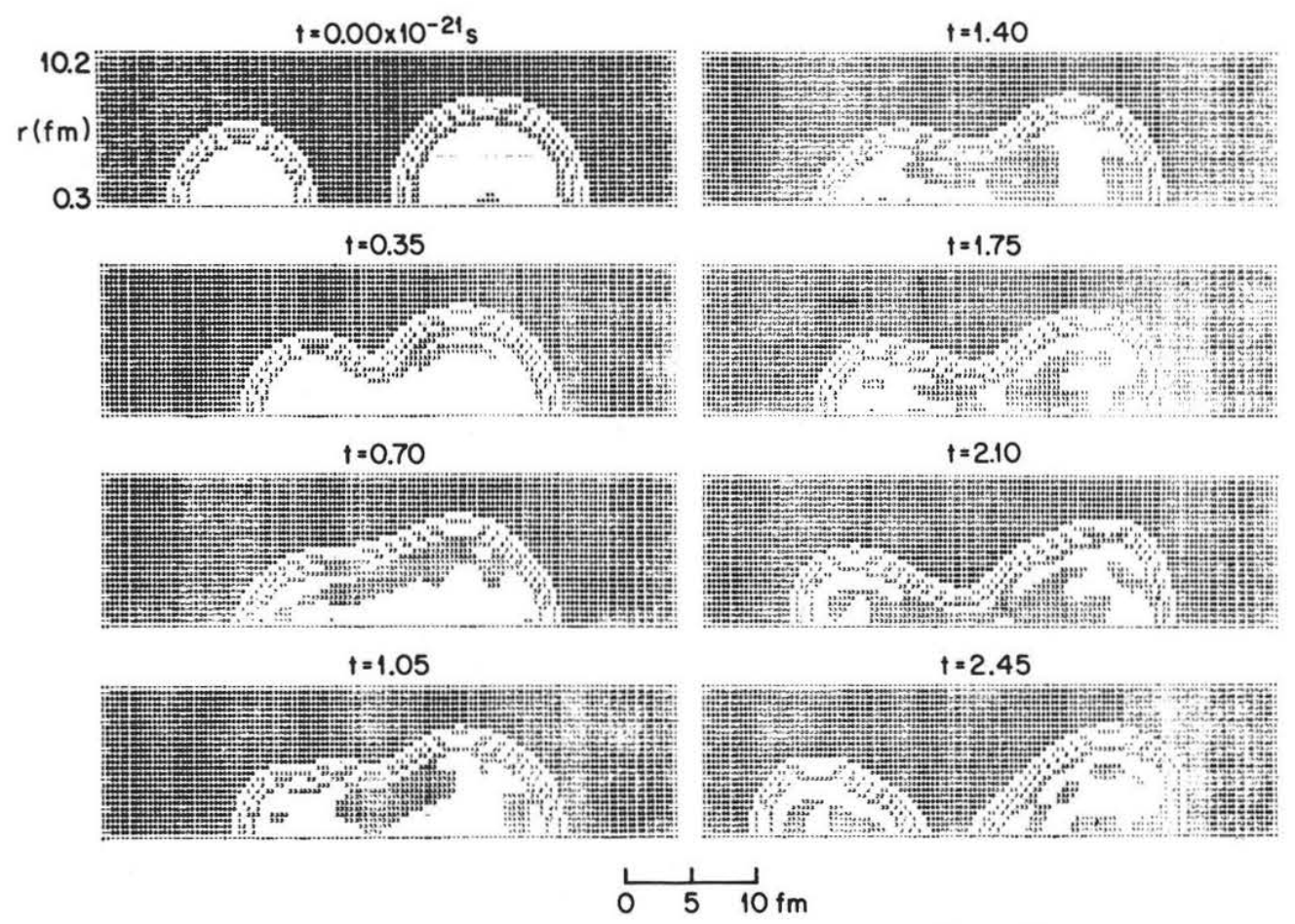

FIG. 2. Equidensity contours in the rotating frame for various times during the ${ }^{84} \mathrm{Kr}+{ }^{209} \mathrm{Bi}$ collision at $E_{\text {lab }}=714 \mathrm{MeV}$ and $l=200$. The mesh parameters are specified in Sec. VIF. 